\title{
The components of the singular locus of a component of a Springer fiber over $x^{2}=0$
}

\author{
Ronit Mansour*and Anna Melnikov \\ Department of Mathematics, University of Haifa, 3498838 Haifa, Israel \\ hadilatil@gmail.com,anna.melnikov@gmail.com
}

\begin{abstract}
For $x \in \operatorname{End}\left(\mathbb{K}^{n}\right)$ satisfying $x^{2}=0$ let $\mathcal{F}_{x}$ be the variety of full flags stable under the action of $x$ (Springer fiber over $x$ ). The full classification of the components of $\mathcal{F}_{x}$ according to their smoothness was provided in 4 in terms of both Young tableaux and link patterns. Moreover in 2 the purely combinatorial algorithm to compute the singular locus of a singular components of $\mathcal{F}_{x}$ is provided. However this algorithm involves the computation of the graph of the component, and the complexity of computations grows very quickly, so that in practice it is impossible to use it. In this paper, we construct another algorithm, derived from the algorithm of Fresse 2, providing all the components of the singular locus of a singular component of $\mathcal{F}_{x}$ in terms of link patterns constructed straightforwardly from its link pattern.
\end{abstract}

\section{Introduction}

Throughout this paper, we set $\mathbb{K}$ to be an algebraically closed field of arbitrary characteristic. We set $V$ to be a vector space of finite dimension $n$. A complete flag of $V$ is a chain $V_{0} \subset V_{1} \subset \cdots \subset V_{n}$ of subspaces of $V$ with $\operatorname{dim} V_{i}=i$ for all $i=0,1, \ldots, n$. We denote the set of all the complete flags by $\mathcal{F}$.

\subsection{Springer fibers and their components}

Let $x$ be a nilpotent endomorphism of $V$. A Springer fiber $\mathcal{F}_{x}$ is a subset of $x$-stable complete flags, that is, flags $\left(V_{0}, V_{1}, \ldots, V_{n}\right)$ such that $x\left(V_{i}\right) \subset V_{i-1}$ for all $i=1,2, \ldots, n$. Clearly, $\mathcal{F}_{x}$ is a closed subvariety of $\mathcal{F}$ (see [11]) and depends on the Jordan form of $x$ only. Note that $\mathcal{F}_{x}$ is reducible unless $x$ is zero or regular. Different aspects of Springer fibers were studied by many authors. In particular many aspects of the study of Springer fibers and its connection to Schubert varieties is described in the survey [14].

The main objects of our interest are the irreducible components of a Springer fiber. We concentrate on the case of $x$ satisfying $x^{2}=0$. In this case the components are described in terms of link

*Supported by ISF grant 797/14 
patterns. We provide the algorithm describing all components of the singular locus of a singular component in terms of admissible pairs of a link pattern.

\subsection{Parametrization of the irreducible components of $\mathcal{F}_{x}$ by Young tableaux}

A nilpotent endomorphism $x: V \mapsto V$ has a unique eigenvalue 0 , so its Jordan form can be written as the list of lengths of its Jordan blocks and since Jordan form is unique up to the order of Jordan blocks this list can be viewed as a partition of $n$. Put $\lambda(x):=\lambda=\left(\lambda_{1}, \ldots, \lambda_{r}\right) \vdash n$ to be this partition. A Young diagram $Y(x)=Y_{\lambda}$ is an array of $r$ rows of boxes starting on the left, with the $i$-th row containing $\lambda_{i}$ boxes. For $\lambda=\left(\lambda_{1}, \ldots, \lambda_{l}\right) \vdash n$ and $\mu=\left(\mu_{1}, \ldots, \mu_{k}\right) \vdash m$ where $m<n$ we say that $\mu$ is a subdiagram of $\lambda$ if $k \leq l$ and for each $i: 1 \leq i \leq k$ one has $\mu_{i} \leq \lambda_{i}$.

Let $\lambda^{*}$ denote the conjugate partition of $\lambda$ that is the list of the lengths of columns in $Y_{\lambda}$. By [12, Sec. II, 5.5], the dimension of $\mathcal{F}_{x}$ is given by $\operatorname{dim} \mathcal{F}_{x}=\sum_{\mu_{i} \in \lambda^{*}(x)}\left(\begin{array}{c}\mu_{i} \\ 2\end{array}\right)$, and in particular for $\lambda=\left(2^{k}, 1^{n-2 k}\right)$ one has $\operatorname{dim} \mathcal{F}=\left(\begin{array}{c}n-k \\ 2\end{array}\right)+\left(\begin{array}{c}k \\ 2\end{array}\right)$.

Fill the boxes of $Y(x)$ with numbers $1,2, \ldots, n$ in such a way that numbers increase from left to right in the rows and from top to bottom in the columns. Such an array is called a standard Young tableau of shape $Y(x)$. We call it a tableau in what follows. We denote the set of all standard tableaux of shape $Y_{\lambda}$ by $T a b_{\lambda}$. Given $T \in T a b_{\lambda}$ where $\lambda \vdash n$, put $T_{\{i\}}$ to be a subtableau of $T$ containing the entries $1,2, \ldots, i$, and respectively put $Y_{i}(T)$ to be its shape.

The components of $\mathcal{F}_{x}$ are parametrized by standard tableaux of shape $Y(x)$, and we provide one of the possible ways of parametrization, following [12. Let $F=\left(V_{0}, \ldots, V_{n}\right) \in \mathcal{F}_{x}$, where $V_{i}$ is $x$-stable subspace of $V$. Thus, the restriction $\left.x\right|_{V_{i}}$ is a nilpotent endomorphism and its Jordan form is represented by a shape $Y\left(\left.x\right|_{V_{i}}\right)$, which is a subdiagram of $Y\left(\left.x\right|_{V_{i+1}}\right)$ (and of $Y(x)$ ). Define

$$
\mathcal{V}_{x}^{T}=\left\{\left(V_{0}, \ldots, V_{n}\right) \in \mathcal{F}_{x} \mid Y\left(\left.x\right|_{V_{i}}\right)=Y_{i}(T), i=1,2, \ldots, n\right\},
$$

which is a partition of $\mathcal{F}_{x}$, namely, $\mathcal{F}_{x}=\coprod_{T \in T a b_{\lambda}} \mathcal{V}_{x}^{T}$. By [12, Sec. II.5.4-5], the set $\mathcal{V}_{x}^{T}$ is a locally closed, irreducible subset of $\mathcal{F}_{x}$ and $\operatorname{dim} \mathcal{V}_{x}^{T}=\operatorname{dim} \mathcal{F}_{x}$. Define $\mathcal{F}_{T}=\overline{\mathcal{V}_{x}^{T}}$ to be the closure in Zariski topology. Then $\left\{\mathcal{F}_{T}\right\}_{T \in T a b_{\lambda}}$ are all the irreducible components of $\mathcal{F}_{x}$. This parametrization carries a lot of information on the components.

\subsection{Smooth and singular components of $\mathcal{F}_{x}$ in general and in case $x^{2}=0$}

Each $\mathcal{F}_{x}$ has at least one smooth component. Moreover, as it is shown in 3 all the components of $\mathcal{F}_{x}$ are smooth if and only if $\lambda=\left(\lambda_{1}, n-\lambda_{1}\right)$ or $\lambda=\left(\lambda_{1}, n-\lambda_{1}-1,1\right)$ or $\lambda=\left(\lambda_{1}, 1^{n-\lambda_{1}}\right.$ ) (or $\left.\lambda=\left(2^{3}\right)\right)$.

In all other cases there is at least one singular component, but in general the classification of the components according to their smoothness is a very difficult problem which is beyond our means. However, the nice exception is the case $x^{2}=0$. Here a full classification of smooth components exists.

In this case all the block in Jordan form of $x$ are of length at most 2. Moreover the number of blocks of length 2 is equal to Rank $x$ so that the components of $\mathcal{F}_{x}$ are parameterized by Young tableaux of shape $\left(2^{k}, 1^{n-2 k}\right)$ where $k=\operatorname{Rank} x$. 
For $T \in T a b_{\lambda}$ where $\lambda \vdash n$ and $i: 1 \leq i \leq n$ let $\operatorname{col}(i)$ be the number of column $i$ belongs to. Put

$$
\tau^{*}(T):=\{i: \operatorname{col}(i+1)>\operatorname{col}(i)\} .
$$

For $T \in T a b_{\left(2^{k}, 1^{n-2 k}\right)}$ let $T_{1}$ denote the first column of $T$ and $T_{2}$ denote the second column of $T$. In this case $\tau^{*}(T)=\left\{i \in T_{1}: i+1 \in T_{2}\right\}$.

For a (finite) set $S$ put $|S|$ to be its cardinality.

For $T \in T a b_{\left(2^{k}, 1^{n-2 k}\right)}$ let $T_{1}=\left(1=a_{1}, \ldots, a_{n-k}\right)$ and $T_{2}=\left(b_{1}, \ldots, b_{k}\right)$. Put

$$
\rho(T):=\left\{\begin{array}{cc}
\left|\tau^{*}(T)\right|+2, & \text { if } a_{n-k}=n \text { and } b_{i}>2 i \forall i: 1 \leq i \leq k ; \\
\left|\tau^{*}(T)\right|+1, & \text { if either }\left(a_{n-k}=n \text { and } \exists i: b_{i}=2 i\right) \text { or } \\
& \quad\left(b_{k}=n \text { and } b_{i}>2 i \forall 1 \leq i \leq k-1\right) ; \\
\left|\tau^{*}(T)\right|, & \text { if } b_{k}=n \text { and } \exists i: 1 \leq i \leq k-1 \text { s.t. } b_{i}=2 i .
\end{array}\right.
$$

For instance, if

$$
T=\begin{array}{|c|c|}
\hline 1 & 4 \\
\hline 2 & 5 \\
\hline 3 & 9 \\
\hline 6 & 10 \\
\cline { 1 - 1 } 7 & \multicolumn{1}{|c}{8} \\
\cline { 1 - 1 } 8 & \multicolumn{1}{|c}{}
\end{array}
$$

then $\tau^{*}(T)=\{3,8\}$, so that $\left|\tau^{*}(T)\right|=2$. Note that $b_{4}=10, b_{1}=4>2, b_{2}=5>4$ and $b_{3}=9>6$. Hence, $\rho(T)=\left|\tau^{*}(T)\right|+1=3$.

For $T \in T a b_{\left(2^{k}, 1^{n-2 k}\right)}$ the simple criteria for $\mathcal{F}_{T}$ to be singular is provided in terms of $\rho(T)$ by [4, Theorem 1.2]:

Theorem 1. For $T \in T a b_{\left(2^{k}, 1^{n-k}\right)} \mathcal{F}_{T}$ is smooth if and only if $\rho(T) \leq 3$.

\subsection{The orbits of centralizer of $x$ for $x^{2}=0$ and link patterns}

In order to study the components of $\mathcal{F}_{x}$ we have to introduce $Z_{x}$-orbits.

Let $G L(V)$ be the general linear group of $V$. Define $Z_{x}=\left\{g \in G L(V) \mid g x g^{-1}=x\right\}$ to be the centralizer of $x$. So, for $g \in Z_{x}$ and $F \in \mathcal{F}_{x}$ one has $x g(F)=g x(F)$ that is $g(F) \in \mathcal{F}_{x}$ so that $Z_{x}$ naturally acts on the complete flags and leaves the Springer fiber $\mathcal{F}_{x}$ stable. However, $\mathcal{F}_{x}$ in general contains an infinite number of $Z_{x}$-orbits. The case $x^{2}=0$ is one of a 3 general cases where $\mathcal{F}_{x}$ is a union of finite number of $Z_{x}$-orbits (the other two cases are $\lambda=\left(\lambda_{1}, 1^{n-\lambda_{1}}\right)$ where all the components are smooth and $\lambda=\left(3,2^{k}, 1^{n-3-2 k}\right)$ in which classification of the components according to their smoothness is far beyond our means).

For $x$ satisfying $x^{2}=0$ and $\operatorname{Rank} x=k Z_{x}$-orbits in $\mathcal{F}_{x}$ are labeled by involutions of symmetric group $S_{n}$ with $k$ disjoint 2-cycles as follows: 
Let $I_{n}$ denote the set of all involutions in $S_{n}$, that is, $I_{n}=\left\{\sigma \in S_{n} \mid \sigma^{2}=i d\right\}$. Let $I_{n, k}$ denote the set of all involutions in $S_{n}$ with $k$ disjoint 2-cycles, that is

$$
I_{n, k}=\left\{\left(i_{1}, j_{1}\right) \ldots\left(i_{k}, j_{k}\right) \in I_{n}: 1 \leq i_{s}<j_{s} \leq n,\left\{i_{s}, j_{s}\right\} \cap\left\{i_{t}, j_{t}\right\}=\emptyset, 1 \leq s \neq t \leq k\right\} .
$$

For $\sigma \in I_{n, k}$, a $\sigma$-basis $\left(v_{1}, \ldots, v_{n}\right)$ of $V$ is a basis of $V$ such that

$$
x\left(v_{i}\right)= \begin{cases}v_{\sigma(i)} & \text { if } \sigma(i)<i \\ 0 & \text { otherwise }\end{cases}
$$

For example, let $\left\{e_{1}, \ldots, e_{5}\right\}$ be a standard basis of $V$ and assume that $x$ with $\lambda(x)=(2,2,1)$ is defined by

$$
x\left(e_{i}\right)= \begin{cases}e_{i-1} & \text { if } i=2,4 \\ 0 & \text { otherwise. }\end{cases}
$$

Let $\sigma=(1,5)(2,3)$ then some $\sigma$-bases are $\left\{e_{1}, e_{3}, e_{4}, e_{5}, e_{2}\right\},\left\{e_{3}, e_{1}, e_{2}, e_{5}+e_{3}, e_{4}\right\},\left\{e_{1}+e_{3}, e_{1}-\right.$ $\left.2 e_{3}, e_{2}-2 e_{4}, e_{5}+e_{1}, e_{2}+e_{4}\right\}$ etc.

A $\sigma$-flag in $\mathcal{F}$ is a complete flag of the form $F=\left(\operatorname{Span}\left\{v_{1}, \ldots, v_{i}\right\}_{i=0}^{n}\right)$, where $\left(v_{1}, \ldots, v_{n}\right)$ is a $\sigma$-basis. We denote the set of all $\sigma$-flags by $\mathcal{Z}_{\sigma}$. Clearly, $\mathcal{Z}_{\sigma} \subset \mathcal{F}_{x}$ and it is $Z_{x}$-orbit of some $\sigma$-flag. By [2] for $x$ of square zero and $\operatorname{Rank} x=k$ one has

$$
\mathcal{F}_{x}=\coprod_{\sigma \in I_{n, k}} \mathcal{Z}_{\sigma}
$$

Since by the result above each $\mathcal{F}_{T}$ is the union of finite number of $Z_{x}$-orbits, in particular there exists a unique $\sigma_{T} \in I_{n, k}$ such that $\mathcal{F}_{T}=\overline{\mathcal{Z}}_{\sigma_{T}}$. We use the combinatorics of $I_{n, k}$ in order to describe singular locus of singular $\mathcal{F}_{T}$ in terms of $\mathcal{Z}_{\sigma}$. A general algorithm for computing the singular locus is provided in [2]. To do this Fresse constructs a graph $G_{T}$, its vertexes are $\left\{\sigma \in I_{n, k}: \mathcal{Z}_{\sigma} \subset \mathcal{F}_{T}\right\}$ (the combinatorial description of vertexes of $G_{T}$ is known (cf. 2.1 for details)) and an edge connects two vertexes if one of them is obtained from another by so called elementary move (also defined combinatorially (cf. 2.2). By 2] $\mathcal{Z}_{\omega}$ is in the singular locus of $\mathcal{F}_{T}$ if and only if in $G_{T}$ the number of edges $\left\{\omega, \omega^{\prime}\right\} \in G_{T}$ is bigger than the number of edges $\left\{\sigma_{T}, v\right\} \in G_{T}$. As one can see at Figure 3 already for the first singular component (case $\lambda=\left(2^{2}, 1^{2}\right)$ ) graph $G_{T}$ is big and complex.

\subsection{The algorithm for the components of a singular locus of $\mathcal{F}_{T}$}

In the paper we construct another algorithm, based on admissible pairs of $\sigma_{T}$ on an interval. We do not use $G_{T}$ and construct all the $\omega_{i}$ such that $\left\{\mathcal{Z}_{\omega_{i}}\right\}_{i=1}^{s}$ are the components of the singular locus of $\mathcal{F}_{T}$ straightforwardly from $\sigma_{T}$. For each pair of minimal $\operatorname{arcs}(i, i+1),(j, j+1)$ in $\sigma_{T}$ satisfying special conditions we construct a component of the singular locus. Since we need more notation in order to explain the algorithm, the exact formula is provided in 3.2 Using this algorithm we show in particular that singular locus of $\mathcal{F}_{T}$ is irreducible iff $\rho(T)=4$. In the subsequent paper we construct a natural stratification of $\mathcal{F}_{T}$ with $\rho(T)=4$ and show that if $\rho(T)>5$ there is no well defined stratification.

The structure of the paper is as follows. In $\S 2$ we provide all the notation and facts needed in what follows in order to make the paper self contained. Then is $\S 3$ we formulate the main theorem and explain its proof. In $\S 4$ we provide all the technical details of the proof. Finally in $\S 5$ we deduce the first results following from our algorithm. The list of notation is provided at the end of the paper. 


\section{Preliminaries}

\subsection{Link patterns}

For $\sigma=\left(i_{1}, j_{1}\right) \ldots\left(i_{k}, j_{k}\right) \in I_{n, k}$, its link pattern $P_{\sigma}$ is a graph with vertices $1,2, \ldots, n$ on a horizontal line and $k$ edges $(\operatorname{arcs})$ connecting $i_{s}$ and $j_{s}$, for all $s=1,2, \ldots, k$. From now and on, we identify the involution $\sigma$ with its link pattern $P_{\sigma}$ and write $\sigma$ for both of them. We set $|\sigma|=k$ to be the number of arcs in $\sigma$.

For $\sigma \in I_{n, k}$, the point $i, 1 \leq i \leq n$, is called a fixed point if $\sigma(i)=i$ (the degree of the vertex $i$ in $\sigma$ is zero), and an end point if $\sigma(i) \neq i$ (the degree of the vertex $i$ in $\sigma$ is one). In the later case, if $\sigma(i)>i, i$ is called a left end point and if $\sigma(i)<i$ it is called a right end point. We denote the set of all fixed points, left end points and right end points of $\sigma$, by $\sigma^{0}, \sigma^{\ell}$ and $\sigma^{r}$, respectively, that is, $\sigma^{0}=\{i: i=\sigma(i)\}, \sigma^{\ell}=\{i: i<\sigma(i)\}$ and $\sigma^{r}=\{i: i>\sigma(i)\}$. We say $(i, j) \in \sigma$ if $i<j$ and $\sigma(i)=j$. For example, Figure 1 presents the link pattern $\sigma=(2,6)(3,8)(4,5) \in I_{9,3}$. Note that $1,7,9 \in \sigma^{0}, 2,3,4 \in \sigma^{\ell}$ and $5,6,8 \in \sigma^{r}$.

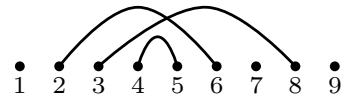

Figure 1: The graph of $(2,6)(3,8)(4,5) \in I_{9,3}$

Let $\sigma$ be any link pattern. We say that $\sigma$ has a crossing if there is a pair of $\operatorname{arcs}(i, j),\left(i^{\prime}, j^{\prime}\right) \in \sigma$ such that $i<i^{\prime}<j<j^{\prime}$, we say that $\left(i^{\prime}, j^{\prime}\right)$ crosses $(i, j)$ on the right and $(i, j)$ crosses $\left(i^{\prime}, j^{\prime}\right)$ on the left in that case. For $(i, j) \in \sigma$, let $c_{\sigma}^{r}((i, j)):=\left|\left\{\left(i^{\prime}, j^{\prime}\right) \in \sigma: i<i^{\prime}<j<j^{\prime}\right\}\right|$ (that is the number of arcs crossing $(i, j)$ on the right) and respectively $c_{\sigma}^{l}((i, j)):=\mid\left\{\left(i^{\prime}, j^{\prime}\right) \in \sigma: i^{\prime}<\right.$ $\left.i<j^{\prime}<j\right\} \mid$ (that is the number of arcs crossing $(i, j)$ on the left). We put $c(\sigma)$ to be the total number of crossings in $\sigma$. Obviously,

$$
c(\sigma)=\sum_{(i, j) \in \sigma} c_{\sigma}^{r}(i, j)=\sum_{(i, j) \in \sigma} c_{\sigma}^{l}(i, j) .
$$

For instance, if $\sigma=(2,6)(3,8)(4,5) \in I_{9,3}$, see Figure 1, then $c(\sigma)=1$, namely, the arc $(2,6)$ crosses the arc $(3,8)$.

For $a<b \in \mathbb{N}$ let $[a, b]:=\{i\}_{i \in \mathbb{N}, a \leq i \leq b}$ denote the set of integer points of interval $[a, b]$.

We call the $\operatorname{arc}(i, j) \in \sigma$ a bridge over $p \in \sigma^{0}$ if $i<p<j$. For $(i, j) \in \sigma$, put $b_{\sigma}((i, j)):=\left|\sigma^{0} \cap[i, j]\right|$ (that is number of $p \in \sigma^{0}$ such that $(i, j)$ is a bridge over them). Set $b(\sigma)$ to be the sum of number of bridges over all fixed points. Obviously,

$$
b(\sigma)=\sum_{(i, j) \in \sigma} b_{\sigma}((i, j)) .
$$

For $f \in \sigma^{0}$ we also introduce for the future use notation $b_{\sigma}(f)$ - the number of bridges over $f$. Obviously $b(\sigma)=\sum_{f \in \sigma^{0}} b_{\sigma}(f)$. For instance, if $\sigma=(2,6)(3,8)(4,5) \in I_{9,3}$, see Figure 11, then $b(\sigma)=1$, namely, the arc $(3,8)$ is a bridge over the fixed point 7 . 
For $a<b \in \mathbb{N}$ let $S_{[a, b]}$ denote the symmetric group on $\{a, a+1, \ldots, b\}$. For $1 \leq a<b \leq n$ and $(i, j) \in \sigma$, we say that $(i, j) \in[a, b]$ if $a \leq i, j \leq b$. Let $\pi_{a, b}(\sigma):=\{(i, j) \in \sigma \cap[a, b]\}$ and we consider it as an involution of $S_{[a, b]}$. Put $R_{[a, b]}(\sigma):=\left|\pi_{a, b}(\sigma)\right|$. Let $R(\sigma) \in M_{a t}\left(\mathbb{Z}^{+}\right)$be the corresponding (strictly upper triangular $n \times n$ matrix) defined by

$$
(R(\sigma))_{i, j}:= \begin{cases}R_{[i, j]}(\sigma) & \text { if } i<j ; \\ 0 & \text { otherwise }\end{cases}
$$

For instance, if $\sigma=(1,4)(2,5) \in I_{5}$ then

$$
R(\sigma)=\left(\begin{array}{ccccc}
0 & 0 & 0 & 1 & 2 \\
0 & 0 & 0 & 0 & 1 \\
0 & 0 & 0 & 0 & 0 \\
0 & 0 & 0 & 0 & 0 \\
0 & 0 & 0 & 0 & 0
\end{array}\right) .
$$

We define a partial order on $\operatorname{Mat}_{n}\left(\mathbb{Z}^{+}\right)$by putting $A \leq B$ for $A, B \in \operatorname{Mat}_{n}\left(\mathbb{Z}^{+}\right)$if for any $1 \leq i, j \leq n$ one has $A_{i, j} \leq B_{i, j}$. This partial order induces partial order on $I_{n}$ as follows. Let $\sigma, v \in I_{n}$ put $\sigma \geq v$ if $R(\sigma) \geq R(v)$.

Recall that for a partially ordered set $(S, \leq)$ the cover of $a \in S$ is

$$
\operatorname{Cov}(a):=\{b \in S: b<a \text { and } a \leq c \leq b \Rightarrow(c=a \text { or } c=b)\} .
$$

For instance,

$$
I_{5,2}=\left\{\begin{array}{l}
(1,2)(3,4),(1,2)(3,5),(1,2)(4,5),(1,3)(2,4),(1,3)(2,5),(1,3)(4,5), \\
(1,4)(2,3),(1,4)(2,5),(1,4)(3,5),(1,5)(2,3),(1,5)(2,4),(1,5)(3,4)
\end{array}\right\} .
$$

The corresponding graph of link patterns is given in Figure2 2 where the first row consists of maximal link patterns and the last row contains the unique minimal link pattern of $I_{5,2}$. Edges between link patterns show the cover of a given link pattern in our relation.

Put $\mathcal{F}_{\sigma}:=\overline{\mathcal{Z}_{\sigma}}$. By $[4$ and [10] one has:

Theorem 2. Let $x \in \operatorname{End}(V)$ with $x^{2}=0$ and Rank $x=k$. Then

- the map $\sigma \mapsto \mathcal{Z}_{\sigma}$ is a bijection between $I_{n, k}$ and the set of $Z_{x}$-orbits of the Springer fiber $\mathcal{F}_{x}$.

- for $\sigma \in I_{n, k}$, the dimension of $\mathcal{F}_{\sigma}$ is given by $\left(\begin{array}{c}n-k \\ 2\end{array}\right)+\left(\begin{array}{c}k \\ 2\end{array}\right)-b(\pi)-c(\pi)$. In particular, the irreducible components of $\mathcal{F}_{x}$ are $\mathcal{F}_{\sigma}$ corresponding to $\sigma \in I_{n, k}$ without crossings and fixed points under an arc.

- for any $\sigma, v \in I_{n, k}, \mathcal{F}_{\sigma} \supset \mathcal{F}_{v}$ if and only if $\sigma \geq v$.

In particular $\mathcal{Z}_{\omega_{o}}$, where $\omega_{o}=(1, n-k+1)(2, n-k+2) \cdots(k, n)$, is the unique closed $Z_{x}$-orbit of $\mathcal{F}_{x}$ (so that $\mathcal{F}_{\omega_{o}}=\mathcal{Z}_{\omega_{o}}$ ) and $\operatorname{dim} \mathcal{F}_{\omega_{o}}=\left(\begin{array}{c}n-2 k \\ 2\end{array}\right)+\left(\begin{array}{c}k \\ 2\end{array}\right)$ and this is the unique element of $I_{n, k}$ satisfying $\omega_{o} \leq \sigma$ for any $\sigma \in I_{n, k}$. 


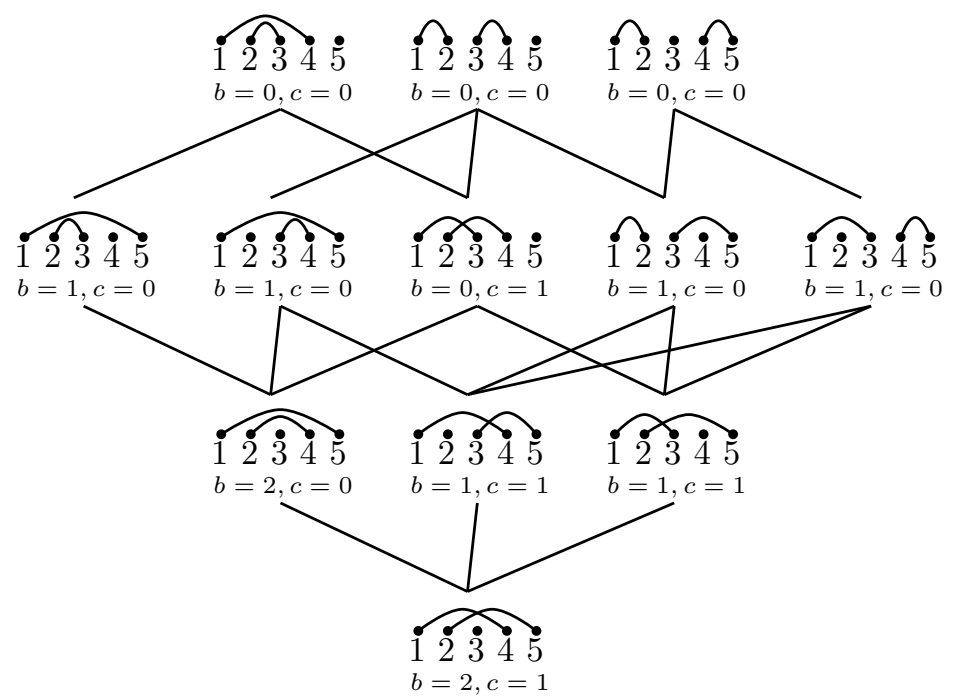

Figure 2: Partial order relation of the link patterns of $I_{5,2}$

By this theorem and [13] $\sigma^{\prime} \in \operatorname{Cov}(\sigma)$ iff $\operatorname{codim}_{\mathcal{F}_{\sigma}} \mathcal{Z}_{\sigma^{\prime}}=1$.

Define $d_{0}:=\operatorname{dim} \mathcal{F}_{\omega_{o}}=\left(\begin{array}{c}n-2 k \\ 2\end{array}\right)+\left(\begin{array}{c}k \\ 2\end{array}\right)$. Note that by the theorem each $\mathcal{F}_{T}$ contains the unique dense $Z_{x}$-orbit $\mathcal{Z}_{\sigma}$, so that there is a bijection between the components of $\mathcal{F}_{x}$ and involutions $\sigma$ without crossings and fixed points under an arc. For $T=\left(T_{1}, T_{2}\right) \in T a b_{\left(2^{k}, 1^{n-2 k}\right)}$ let $T_{1}=\left(1, a_{2}, \ldots, a_{n-k}\right)$ denote the first column of $T$ and $T_{2}=\left(b_{1}, \ldots, b_{k}\right)$ denote its second column. $\sigma_{T}$ such that $\mathcal{F}_{T}=\mathcal{F}_{\sigma_{T}}$ is constructed as follows. We define

$$
\sigma_{T}=\left(i_{1}, b_{1}\right), \ldots,\left(i_{k}, b_{k}\right),
$$

where $i_{1}=b_{1}-1$ and $i_{s}=\max \left\{a_{m} \in T_{1} \backslash\left\{i_{1}, \ldots, i_{s-1}\right\}: a_{m}<b_{s}\right\}$.

For instance, let

$$
T=\begin{array}{|c|c|}
\hline 1 & 4 \\
\hline 2 & 5 \\
\hline 3 & 9 \\
\hline 6 & 10 \\
\cline { 1 - 1 } 7 & \multicolumn{1}{|c}{} \\
\cline { 1 - 1 } 8 & \multicolumn{1}{|c}{} \\
\cline { 1 - 1 }
\end{array}
$$

One has $b_{1}=4, b_{2}=5, b_{3}=9$ and $b_{4}=10$. Thus, $i_{1}=b_{1}-1=3, i_{2}=\max \{a \in\{1,2,6,7,8\} \mid a<$ $5\}=2, i_{3}=\max \{a \in\{1,6,7,8\} \mid a<9\}=8$ and $i_{4}=\max \{a \in\{1,2,6,7\} \mid a<10\}=7$. Thus, $\sigma_{T}=(3,4)(2,5)(8,9)(7,10) \in I_{10,4}$.

Given $\sigma=\left(i_{1}, j_{1}\right) \ldots\left(i_{k}, j_{k}\right) \in I_{(n, k)}$ where $j_{1}<j_{2}<\ldots j_{k}$ without crossings and fixed points under an arc, $T \in \operatorname{Tab}_{\left(2^{k}, 1^{n-2 k}\right)}$ such that $\sigma_{T}=\sigma$ is $T=\left(T_{1}, T_{2}\right)$ where $T_{2}=\left(j_{1}, \ldots, j_{k}\right)$ and $T_{1}=\{i\}_{i=1}^{n} \backslash\left\{j_{s}\right\}_{s=1}^{k}$ written in increasing order. We call $\sigma \in I_{n, k}$ without crossings and fixed point under an arc maximal and denote by $I_{n, k}^{\max }$ the subset of all maximal $\sigma$ in $I_{n, k}$. 
For $\sigma \in I_{n, k}^{\max }$ put $\tau^{*}(\sigma):=\{i:(i, i+1) \in \sigma\}$. One can easily see that $\tau^{*}\left(\sigma_{T}\right)=\tau^{*}(T)$. Respectively, put

$$
\rho(\sigma)= \begin{cases}\left|\tau^{*}(\sigma)\right|+2 & \text { if } 1, n \in \sigma^{0} \\ \left|\tau^{*}(\sigma)\right|+1 & \text { if either }\left|\sigma^{0} \cap\{1, n\}\right|=1, \text { or }(1, n) \in \sigma \\ \left|\tau^{*}(\sigma)\right| & \text { otherwise. }\end{cases}
$$

Again, $\rho\left(\sigma_{T}\right)=\rho(T)$.

\section{$2.2 \quad G_{T}$ graphs and smoothness of $\mathcal{F}_{T}$}

Given $\sigma \in I_{(n, k)}$, for $\left(i_{1}, j_{1}\right), \ldots,\left(i_{s}, j_{s}\right) \in \sigma$ put $\sigma_{\left(i_{1}, j_{1}\right), \ldots,\left(i_{s}, j_{s}\right)}^{-} \in I_{n, k-s}$ to be a link pattern obtained from $\sigma$ by deleting $\operatorname{arcs}\left(i_{1}, j_{1}\right), \ldots,\left(i_{s}, j_{s}\right)$. For $i<j \in \sigma^{0}$ put $(i, j) \sigma=\sigma(i, j) \in I_{n, k+1}$ to be a link pattern obtained from $\sigma$ by adding arc $(i, j)$.

For $(i, j) \in \sigma$ and $f \in \sigma^{0}$ such that $(i, j)$ is bridge over $f$ we say that $\sigma^{\prime} \in I_{n, k}$ is obtained from $\sigma$ by an elementary move forward of first type if either $\sigma^{\prime}=(i, f) \sigma_{(i, j)}^{-}$or $\sigma^{\prime}=(f, j) \sigma_{(i, j)}^{-}$. We say that $\sigma$ is obtained by an elementary move backward of first type from $\sigma^{\prime}$ in this case.

For $(i, j),(\ell, m) \in \sigma$ satisfying $i<\ell<j<m$, we say that $\sigma^{\prime} \in I_{n, k}$ is obtained from $\sigma$ by an elementary move forward of the second type if either $\sigma^{\prime}=(i, \ell)(j, m) \sigma_{(i, j),(\ell, m)}^{-}$or $\sigma^{\prime}=$ $(i, m)(j, \ell) \sigma_{(i, j),(\ell, m)}^{-}$. Again say that $\sigma$ is obtained by an elementary move backward of second type from $\sigma^{\prime}$ in this case.

We call $\sigma^{\prime}$ a predecessor of $\sigma$ if is obtained from $\sigma$ by an elementary move forward. Note that $\sigma^{\prime}>\sigma$.

For $\sigma \in I_{n, k}$, graph $G_{\sigma}$ is a graph on vertices

$$
\mathcal{V}_{G_{\sigma}}=\left\{v \in I_{n, k}: v \leq \sigma\right\}
$$

and with edges

$$
\mathcal{E}_{G_{\sigma}}=\left\{\left(v, v^{\prime}\right): v, v^{\prime} \in \mathcal{V}_{G_{\pi}} \text { and one of them is a predecessor of another }\right\} .
$$

For $v \in \mathcal{V}_{G_{\sigma}}$, let $a_{\sigma, v}$ denote the number of edges of vertex $v$ in $G_{\sigma}$. For instance, Figure 3 presents the graph $G_{\sigma}$, where $\sigma=(2,3)(4,5) \in I_{6,2}$. Column $i$ of the graph contains $\sigma^{\prime}$ with $\operatorname{codim}_{\mathcal{F}_{(2,3)(4,5)}} \mathcal{Z}_{\sigma^{\prime}}=i-1$.

Here we have $a_{(2,3)(4,5),(2,3)(4,5)}=5$ and $a_{(2,3)(4,5),(1,5)(2,6)}=9$. In what follows we will write $v \in G_{\sigma}$ instead of $v \in \mathcal{V}_{G_{\sigma}}$ in order to simplify the notation. Recall that a graph is called $p$-regular if every vertex has $p$ edges. For a projective variety $\mathcal{V}$ and a point $F \in \mathcal{V}$, let $\mathcal{T}_{F}(\mathcal{V})$ denote the tangent space to $\mathcal{V}$ at point $F$. By [2] one has

Theorem 3. Let $x \in \operatorname{End}(V)$ with $x^{2}=0$ and $\operatorname{Rank} x=k$. For $\sigma, v \in I_{n, k}$ with $v \in G_{\sigma}$, let $\mathcal{Z}_{\sigma}, \mathcal{Z}_{v} \subset \mathcal{F}_{x}$ be the corresponding $Z_{x}$-orbits. Let $F_{v} \in \mathcal{Z}_{v}$ denote a point of orbit $\mathcal{Z}_{v}$. Define $p_{\sigma}:=\left(\begin{array}{c}n-k \\ 2\end{array}\right)-\left(\begin{array}{c}n-2 k \\ 2\end{array}\right)-b(\sigma)-c(\sigma)$.

- One has $\operatorname{dim} \mathcal{T}_{F_{v}}\left(\mathcal{F}_{\sigma}\right)=a_{\sigma, v}+d_{0}$. In particular, $a_{\sigma, v} \geq \operatorname{dim} \mathcal{Z}_{\sigma}-d_{0}=a_{\sigma, \sigma}=p_{\sigma}$. 


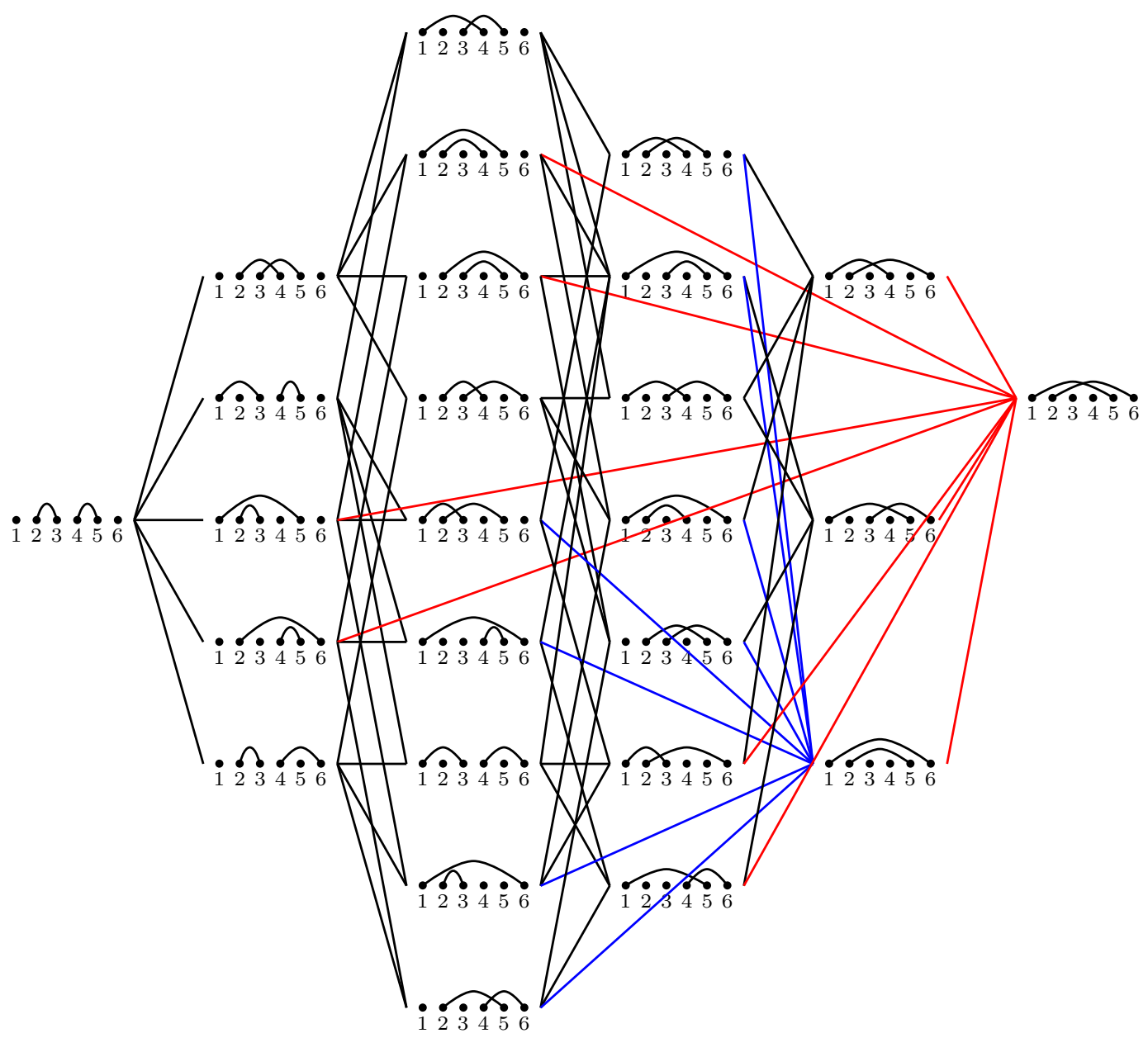

Figure 3: The graph $G_{\sigma}$, where $\sigma=(2,3)(4,5) \in S_{6}$.

- $\mathcal{F}_{\sigma}$ is smooth if and only if the graph $G_{\sigma}$ is $p_{\sigma}$-regular. Otherwise, the singular locus of $\mathcal{F}_{\sigma}$ is $\underset{v \in G_{\sigma}, a_{\sigma, v}>p_{\sigma}}{\mathcal{Z}_{v}}$.

For instance, one can see at Figure 3 that $\mathcal{F}_{(2,3)(4,5)}$ for $n=6$ is singular and its singular locus is $\mathcal{F}_{(1,6)(2,5)}=\mathcal{Z}_{(1,6)(2,5)} \sqcup \mathcal{Z}_{(1,5)(2,6)}$. Indeed, $\operatorname{dim} \mathcal{F}_{(2,3)(4,5)}=7$ and

$$
\operatorname{dim} \mathcal{T}_{F_{(1,6)(2,5)}}\left(\mathcal{F}_{(2,3)(4,5)}\right)=\operatorname{dim} \mathcal{T}_{F_{(1,5)(2,6)}}\left(\mathcal{F}_{(2,3)(4,5)}\right)=11
$$

Note that $\mathcal{F}_{(1,6)(2,5)}=\mathcal{Z}_{(1,6)(2,5)} \sqcup \mathcal{Z}_{(1,5)(2,6)}$ so that $\mathcal{F}_{(1,6)(2,5)}$ is the component of singular locus of $\mathcal{F}_{(2,3)(4,5)}$. 


\section{Components of the singular locus for a given $\mathcal{F}_{\sigma}$}

\subsection{Admissible pair}

Let $\sigma \in I_{n, k}$ and $1 \leq s<t \leq n$, we say that $(i, j),\left(i^{\prime}, j^{\prime}\right) \in \sigma$ is an admissible pair at $[s, t]$ if

• $s<i<j<i^{\prime}<j^{\prime}<t$;

- $s, t \in \pi_{s, t}(\sigma)^{0}$;

- for any $\ell: s<\ell<j$ or $i^{\prime}<\ell<t$ one has $\ell \notin \pi_{s, t}(\sigma)^{0}$, that is additional fixed points can be only at $\left[j, i^{\prime}\right]$;

- for any $(r, q) \in \pi_{s, t}(\sigma)$ such that either $r<i<q$ or $r<j^{\prime}<q$ or $r<j<i^{\prime}<q$ one has $r<i$ and $j^{\prime}<q$, that is, there are no arcs crossing $(i, j)$ or $\left(i^{\prime}, j^{\prime}\right)$ and every $(r, q)$ over either $(i, j)$ or $\left(i^{\prime}, j^{\prime}\right)$ is over both of them;

- for any $(r, q),\left(r^{\prime}, q^{\prime}\right) \in \pi_{s, t}(\sigma)$ satisfying $r^{\prime}<r<i<j^{\prime}<q$, one has $q<q^{\prime}$, that is, all the arcs over both $(i, j)$ and $\left(i^{\prime}, j^{\prime}\right)$ are concentric;

- let $k$ be the number of fixed points at $\left[j, i^{\prime}\right]$; and $r$ the number of arcs over both $(i, j)$ and $\left(i^{\prime}, j^{\prime}\right)$, then $k r=0$.

For $\sigma \in I_{n, k}$ such that $\mathcal{F}_{\sigma}$ is singular let

$$
\operatorname{Sing}(\sigma):=\left\{v: \mathcal{F}_{v} \text { is a component of the signular locus of } \mathcal{F}_{\sigma}\right\} \text {. }
$$

In order to formulate the main theorem we need the notions of maximal completion and concatenation, which we define in the next subsection.

\subsection{Maximal completion, concatenation and the main theorem}

For $\sigma \in I_{n, k}$ let $\sigma_{+a}$ be obtained by moving points $i: 1 \leq i \leq n$ to $i+a$. That is $(i, j) \in \sigma$ is translated to $(i+a, j+a) \in \sigma_{+a}$. We can consider $\sigma_{+a}$ as an element of $I_{n+a, k}$, which means that we add $a$ fixed points to $\sigma$ on the left. We can consider $\sigma$ as an element of $I_{n+a, k}$ by adding $a$ fixed points on the right simply by writing $\sigma \in I_{n+a, k}$.

Definition 4. Given $\sigma \in I_{n, k}$ with $2 k<n$. Let $m_{1}=\min \sigma^{0}$ and $m_{2}=\max \sigma^{0}$. We call $\left(1, m_{1}+\right.$ 1) $\sigma_{+1} \in I_{n+1, k+1}(1,0)$-maximal completion of $\sigma$ and $\left(m_{2}, n+1\right) \sigma \in I_{n+1, k+1}(0,1)$-maximal completion of $\sigma$. If $\widehat{\sigma} \in I_{n+s_{1}+s_{2}, k+s_{1}+s_{2}}$ is obtained from $\sigma$ by series of $s_{1}$ maximal completions on the left and $s_{2}$ maximal completions on the right we call it $\left(s_{1}, s_{2}\right)$-maximal completion.

Note that the order of completion is not important. Since $\sigma \in I_{n, k}$ has $n-2 k$ fixed points $(s, n-2 k-s)$-maximal completions are the largest possible. We will call $\sigma \in I_{2 k, k} k$-complete link patterns.

Definition 5. For $\sigma \in I_{n}$ and $v \in I_{m}$ put $\sigma v_{+n} \in I_{n+m}$ to be their concatenation. It is obtained moving arcs of $v$ by $n$ and "gluing" the new set of arcs to $\sigma$. 
Note that each maximal link pattern is a concatenation of complete maximal link patterns and fixed points between them.

Theorem 6. Consider $\sigma \in I_{n, k}^{\max }$ with $\rho(\sigma) \geq 4$. Let $S=\left\{\left(\left\{(i, j),\left(i^{\prime}, j^{\prime}\right)\right\},[s, t]\right)\right\}$ be the set of all admissible pairs at all possible $[s, t]$. For $x \in S$ let $a=\max \left(\sigma^{0} \cap[1, s]\right)$ if it is not empty, and $a=0$ otherwise and $b=\min \left(\sigma^{0} \cap[t, n]\right)$ if it is not empty and $b=n+1$ otherwise. Let $v=\pi_{s, t}(\sigma)_{(i, j),\left(i^{\prime}, j^{\prime}\right)}^{-}\left(i, j^{\prime}\right)(s, t)$.

(i) If $a=s$ put $v_{l}=\pi_{1, a-1}(\sigma)$ and $l=0$ and $i=a-1$. Otherwise put $v_{l}=\pi_{1, a}(\sigma)$ and $l=s-1-a$ and $i=a$;

(ii) If $b=t$ put $v_{r}=\pi_{b+1, n}(\sigma)$ and $r=0$ and $j=b$. Otherwise put $v_{r}=\pi_{b, n}(\sigma)$ and $r=b-t-1$ and $j=b-1$;

Let $\widehat{v}$ be $(l, r)$-completion of $v$ and $v(x)=v_{l} \widehat{v}_{+i}\left(v_{r}\right)_{+j}$. Then $\operatorname{Sing}(\sigma)=\{v(x)\}_{x \in S}$. Moreover, $\operatorname{Sing}(\sigma)$ contains the unique element if and only if $\rho(\sigma)=4$.

For instance, there are 5 elements in $\operatorname{Sing}(\sigma)$ for $\sigma=(2,3)(4,5)(6,7) \in I_{8,3}^{\max }$, see Figure 4

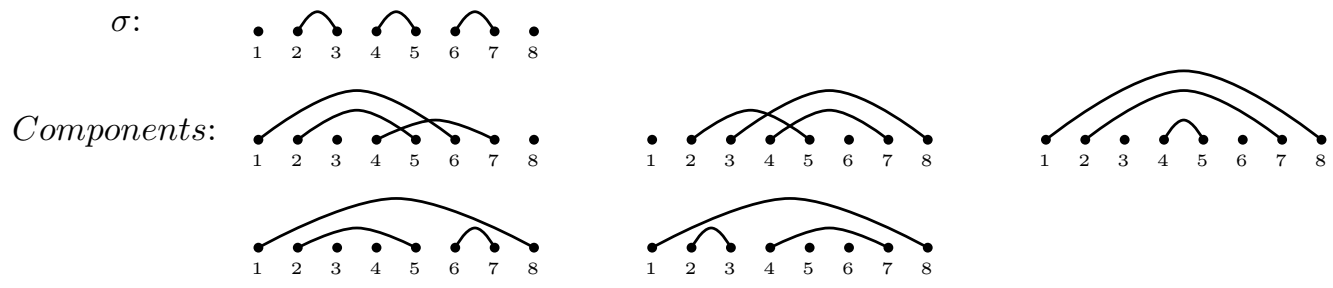

Figure 4: Link patterns $\sigma=(2,3)(4,5)(6,7) \in I_{8,3}^{\max }$ and its components

As we show in Subsection 5 for $\sigma \in I_{n, k}^{\max }$ such that $\rho(\sigma)>4$ the number of elements in $\operatorname{Sing}(\sigma)$ depends not only on $\rho(\sigma)$ but on the structure of $\sigma$, and this happens exactly because the same pair can be admissible at different intervals, depending on the structure of $\sigma$. In particular, if $\rho(\sigma)=5$ then the number of elements in $\operatorname{Sing}(\sigma)$ is between 3 and 5.

To prove this theorem we start in Section 4.1 with construction of $v \in \operatorname{Sing}(\sigma)$ using an admissible pair on $[1, n]$, then in Section 4.2 we show that for $\sigma \in I_{2 k, k}$ a maximal $(s-1, n-t)$-completion of $v \in \operatorname{Sing}\left(\pi_{s, t}(\sigma)\right)$ is in $\operatorname{Sing}(\sigma)$ and that for $\sigma \in I_{n, k}, v \in \operatorname{Sing}(\sigma)$ and $\sigma^{\prime} \in I_{m, k}$ concatenation $v \sigma_{+n}^{\prime} \in \operatorname{Sing}\left(\sigma \sigma_{+n}^{\prime}\right)$ and $\sigma^{\prime} v_{+m} \in \operatorname{Sing}\left(\sigma^{\prime} \sigma_{+m}\right)$. Finally, in Section 4.3 we show that for $\sigma \in I_{n, k}^{\max }$ with $\rho(\sigma) \geq 4, v \in \operatorname{Sing}(\sigma)$ iff it is obtained by the algorithm above.

As an immediate corollary of this result and its proof we get

Corollary 7. For $\sigma \in I_{n, k}^{\max }$ with $\rho(\sigma) \geq 4$ and for $v \in \operatorname{Sing}(\sigma)$, one has

$$
\operatorname{codim}_{\mathcal{F}_{\sigma}} \mathcal{F}_{v} \geq 4 \quad \text { and } \quad \operatorname{dim} \mathcal{T}_{F_{v}}\left(\mathcal{F}_{\sigma}\right)=\operatorname{codim}_{\mathcal{F}_{\sigma}} \mathcal{F}_{v}+\operatorname{dim} \mathcal{F}_{\sigma}
$$




\section{Proof of Theorem 6}

\subsection{Basic case}

Let us first show the following basic case

Theorem 8. Let $\sigma$ be a link pattern such that $(i, j),\left(i^{\prime}, j^{\prime}\right)$ is an admissible pair at $[1, n]$. Put $v:=(1, n)\left(i, j^{\prime}\right) \sigma_{(i, j)\left(i^{\prime}, j^{\prime}\right)}^{-}$. Then $\mathcal{F}_{v}$ is a component of the singular locus of $\mathcal{F}_{\sigma}$.

Graphically one has (where all other points and arcs are drawn by points)
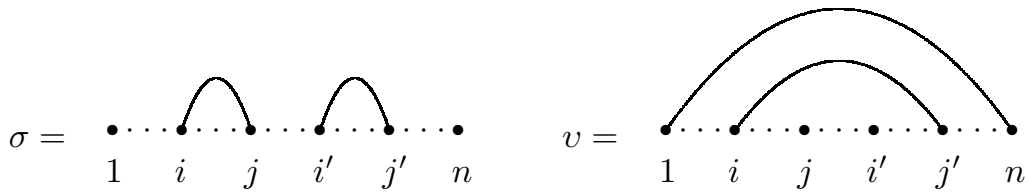

To prove the theorem we show in Proposition 9 below that if $(i, j),\left(i^{\prime}, j^{\prime}\right)$ is an admissible pair then $F_{v}$ is a singular point of $\mathcal{F}_{\sigma}$, and then in Proposition 10 we show that for any $v^{\prime}: v<v^{\prime}<\sigma$ one has $\operatorname{dim} \mathcal{T}_{F_{v}^{\prime}}\left(\mathcal{F}_{\sigma}\right)=\operatorname{dim} \mathcal{F}_{\sigma}$ so that $v \in \operatorname{Sing}(\sigma)$.

Proposition 9. Let $\sigma \in I_{n, k}^{\max }$ be a link pattern such that $(i, j),\left(i^{\prime}, j^{\prime}\right)$ is an admissible pair at $[1, n]$. Put $v=(1, n)\left(i, j^{\prime}\right) \sigma_{(i, j)\left(i^{\prime}, j^{\prime}\right)}^{-}$. Then $F_{v}$ is a singular point of $\mathcal{F}_{\sigma}$. Let

- $k$ be the number of fixed points of $\sigma$ on $\left[j, i^{\prime}\right]$

- $R$ be the set of arcs of $\sigma$ over both $(i, j)$ and $\left(i^{\prime}, j^{\prime}\right)$ that is $R:=\left\{(s, t) \in \sigma: s<i, j^{\prime}<t\right\}$, and $r=|R|$.

Then

$$
\operatorname{dim} \mathcal{T}_{F_{v}}\left(\mathcal{F}_{\sigma}\right)=\operatorname{dim} \mathcal{F}_{\sigma}+(k+1)(2 r+2)+2
$$

Proof. At first, let us show

$$
\operatorname{codim}_{\mathcal{F}_{\sigma}} \mathcal{F}_{v}=4+2(k+r)
$$

- For $(s, t) \in \sigma_{(i, j)\left(i^{\prime}, j^{\prime}\right)}^{-}$one has $c_{v}^{\ell}(s, t)=c_{\sigma}^{\ell}(s, t)$ because there are no arcs crossing $(i, j)$ and $\left(i^{\prime}, j^{\prime}\right)$.

- For $(s, t) \in \sigma_{(i, j)\left(i^{\prime}, j^{\prime}\right)}^{-}$one has

$$
b_{v}(s, t)= \begin{cases}b_{\sigma}(s, t)+2 & \text { if }(s, t) \in R \\ b_{\sigma}(s, t) & \text { otherwise }\end{cases}
$$

- $c_{v}^{\ell}\left(i, j^{\prime}\right)=c_{\sigma}^{\ell}(i, j)=c_{v}^{\ell}(1, n)=0$;

- $b_{v}\left(i, j^{\prime}\right)=b_{v}(1, n)=k+2$ and $b_{\sigma}(i, j)=b_{\sigma}\left(i^{\prime}, j^{\prime}\right)=0$. 
Thus

$$
\begin{aligned}
b(v)+c(v) & =b_{v}\left(i, j^{\prime}\right)+b_{v}(1, n)+\sum_{(s, t) \in \sigma_{(i, j)\left(i^{\prime}, j^{\prime}\right)}^{-}} b_{v}(s, t)+\sum_{(s, t) \in \sigma_{(i, j)\left(i^{\prime}, j^{\prime}\right)}^{-}} c_{v}^{\ell}(s, t) \\
& =2(k+2)+b(\sigma)+2 r+c(\sigma)=b(\sigma)+c(\sigma)+4+2(k+r),
\end{aligned}
$$

which provides $(*)$.

Now, let us compute $\operatorname{dim} \mathcal{T}_{F_{v}}\left(\mathcal{F}_{\sigma}\right)$. In order to do that, we have to find the number of all $\sigma^{\prime}$ such that $v<\sigma^{\prime} \leq \sigma$, where $\sigma^{\prime}$ is a predecessor of $v$. Such $\sigma^{\prime}$ can be obtained by one of the following ways:

(i) $\sigma^{\prime}=v_{(s, t)}^{-}\left(s, t^{\prime}\right)$ where $(s, t) \in\left\{\left(i, j^{\prime}\right),(1, n)\right\} \sqcup R$ and $t^{\prime}$ is any of fixed points of $v$ at $\left[j, i^{\prime}\right]$. The number of such $\sigma^{\prime}$ is

$$
(k+2)(r+2) .
$$

(ii) $\sigma^{\prime}=v_{(s, t)}^{-}\left(s^{\prime}, t\right)$ where $(s, t) \in\left\{\left(i, j^{\prime}\right),(1, n)\right\} \sqcup R$ and $s^{\prime}$ is any of fixed points of $v$ at $\left[j, i^{\prime}\right]$. The number of such $\sigma^{\prime}$ is

$$
(k+2)(r+2) .
$$

These are all predecessors of $v$ which are smaller than $\sigma$. Recall that $\operatorname{dim} \mathcal{T}_{F_{v}}\left(\mathcal{F}_{\sigma}\right)$ is equal to $\operatorname{dim} \mathcal{F}_{v}$ plus the number of predecessors which are smaller than $\sigma$. So we get $\operatorname{dim} \mathcal{T}_{F_{v}}\left(\mathcal{F}_{\sigma}\right)=$ $\operatorname{dim} \mathcal{F}_{v}+(k+2)(2 r+4)$. Hence, taking into account $(*)$ we get

$$
\begin{aligned}
\operatorname{dim} \mathcal{T}_{F_{v}}\left(\mathcal{F}_{\sigma}\right) & =\operatorname{dim} \mathcal{F}_{v}+(k+2)(2 r+4)=\operatorname{dim} F_{\sigma}-(4+2(k+r))+(k+2)(2 r+4) \\
& =\operatorname{dim} \mathcal{F}_{\sigma}+(k+1)(2 r+2)+2 .
\end{aligned}
$$

Therefore, $F_{v}$ is a singular point of $\mathcal{F}_{\sigma}$.

Now let us show

Proposition 10. Let $(i, j),\left(i^{\prime}, j^{\prime}\right) \in \sigma$ be admissible at $[1, n]$. Let

$$
v=\sigma_{(i, j),\left(i^{\prime}, j^{\prime}\right)}^{-}\left(i, j^{\prime}\right)(1, n) .
$$

Then for any $v^{\prime}$ such that $\sigma \geq v^{\prime}>v$ one has $\operatorname{dim} \mathcal{T}_{F_{v^{\prime}}}\left(\mathcal{F}_{\sigma}\right)=\operatorname{dim} \mathcal{F}_{\sigma}$, so that $v \in \operatorname{Sing}(\sigma)$.

Proof. Since for any $v^{\prime}: \sigma \geq v^{\prime}>v$ there exists $v^{\prime \prime}: v^{\prime} \geq v^{\prime \prime}>v$ such that

$$
\operatorname{dim} \mathcal{F}_{v^{\prime \prime}}=\operatorname{dim} \mathcal{F}_{v}+1
$$

and

$$
\operatorname{dim} \mathcal{T}_{F_{v^{\prime}}}\left(\mathcal{F}_{\sigma}\right) \leq \operatorname{dim} \mathcal{T}_{F_{v^{\prime \prime}}}\left(\mathcal{F}_{\sigma}\right)
$$

it is enough to check that the proposition is true only for $v^{\prime}$ predecessors of $v$ such that $\sigma>v^{\prime}$ and $\operatorname{dim} \mathcal{F}_{v^{\prime}}=\operatorname{dim} \mathcal{F}_{v}+1$. 
Since $\left\{(i, j),\left(i^{\prime}, j^{\prime}\right)\right\}$ is admissible at $[1, n]$, there are no fixed points in $v$ outside of $\left[j, i^{\prime}\right]$ so that the minimal fixed point of $v$ is $j$ and the maximal fixed point of $v$ is $i^{\prime}$. Thus, according to the proof of Proposition 9 the predecessors of $v$ smaller than $\sigma$ can be only of types (i) and (ii) namely:

$$
v_{1}=v_{(s, t)}^{-}\left(s, i^{\prime}\right), \quad \text { or } \quad v_{2}=v_{(s, t)}^{-}(j, t)
$$

where $(s, t) \in R \sqcup\left\{\left(i, j^{\prime}\right),(1, n)\right\}$.

Exactly as in the proof of Proposition 9 we have to compare $\operatorname{codim}_{\mathcal{F}_{\sigma}} \mathcal{F}_{v_{m}}$ and $\operatorname{dim} \mathcal{T}_{F_{v_{m}}}\left(\mathcal{F}_{\sigma}\right)$, where $m=1,2$. By $(*)$ in the proof of Proposition 9, we have $\operatorname{codim}_{\mathcal{F}_{\sigma}} \mathcal{F}_{v_{m}}=3+2(k+r)$. The computations of $\operatorname{dim} \mathcal{T}_{F_{v_{m}}}\left(\mathcal{F}_{\sigma}\right)$ are exactly the same for both cases and are very similar to the computations in the proof of Proposition 9. For example, we compute $\operatorname{dim} \mathcal{T}_{v_{1}}\left(\mathcal{F}_{\sigma}\right)$.

We have to compute the number of all $\sigma^{\prime}: \sigma \geq \sigma^{\prime}$, where $\sigma^{\prime}$ is a predecessor of $v_{1}$. They are obtained as follows:

- $\sigma^{\prime}=\left(v_{1}\right)_{\left(s, i^{\prime}\right)}^{-}(s, f)$ for $f$ a fixed point of $\left[j, i^{\prime}-1\right]$. The number of such $\sigma^{\prime}$ is $k+1$.

- $\sigma^{\prime}=\left(v_{1}\right)_{\left(s^{\prime}, t^{\prime}\right)}^{-}\left(f, t^{\prime}\right)$ for $f$ a fixed point of $\left[j, i^{\prime}-1\right]$ and

$$
\left(s^{\prime}, t^{\prime}\right) \in\left\{(1, n),\left(i, j^{\prime}\right)\right\} \sqcup R \backslash\{(s, t)\} .
$$

The number of such $\sigma^{\prime}$ is $(k+1)(r+1)$.

- $\sigma^{\prime}=\left(v_{1}\right)_{\left(s^{\prime}, t^{\prime}\right),\left(s, i^{\prime}\right)}^{-}\left(s^{\prime}, i^{\prime}\right)\left(s, t^{\prime}\right)$ for $\left(s^{\prime}, t^{\prime}\right) \in\left\{(1, n),\left(i, j^{\prime}\right)\right\} \sqcup R$ such that $s^{\prime}>s$. Let us denote the number of such $\sigma^{\prime}$ by $a$.

- $\sigma^{\prime}=\left(v_{1}\right)_{\left(s^{\prime}, t^{\prime}\right)}^{-}\left(s^{\prime}, t\right)$ for $\left(s^{\prime}, t^{\prime}\right) \in\left\{(1, n),\left(i, j^{\prime}\right)\right\} \sqcup R$ such that $t^{\prime}>t$. Note that since all the arcs of $R$ are concentric we get that $\left(s^{\prime}, t^{\prime}\right)$ satisfies $s^{\prime}<s$, so that the number of such $\sigma^{\prime}$ is $r+1-a$.

- Finally, note that for $v^{\prime}=\left(v_{1}\right)_{\left(s, i^{\prime}\right)}^{-}\left(f, i^{\prime}\right)$ or $v^{\prime}=\left(v_{1}\right)_{\left(s^{\prime}, t^{\prime}\right)}^{-}\left(s^{\prime}, f\right)$ for $f \in\left[j, i^{\prime}-1\right]$ and $\left(s^{\prime}, t^{\prime}\right) \in\left\{(1, n),\left(i, j^{\prime}\right)\right\} \sqcup R$ such that $s^{\prime} \neq s$ one has $v^{\prime} \nless \sigma$.

All other predecessors also provide us with $\sigma^{\prime} \nless \sigma$.

All together this shows that

$$
\begin{aligned}
\operatorname{dim} \mathcal{T}_{F_{v_{1}}}\left(\mathcal{F}_{\sigma}\right) & =\operatorname{dim} \mathcal{F}_{v_{1}}+(k+1)+(k+1)(r+1)+a+r+1-a=\operatorname{dim} \mathcal{F}_{v_{1}}+(k+1)(r+2)+r+1 \\
& =\operatorname{dim} \mathcal{F}_{v_{1}}+(r+2)(k+2)-1= \begin{cases}\operatorname{dim} \mathcal{F}_{v_{1}}+2(r+k)+3 & \text { if } r k=0 ; \\
\operatorname{dim} \mathcal{F}_{v_{1}}+2(r+k)+3+k r & \text { otherwise; }\end{cases}
\end{aligned}
$$

So we get that $v \in \operatorname{Sing}(\sigma)$ if and only if $k r=0$ (here we used the last condition of admissibility).

\subsection{Construction of $v \in \operatorname{Sing}(\sigma)$ from $v\left((i, j),\left(i^{\prime}, j^{\prime}\right),[s, t]\right)$}

For $\sigma \in I_{n, k}$ where $n>2 k$ let $\widehat{\sigma}$ be either $(1,0)$ or $(0,1)$ maximal completion of $\sigma$. We show that $v \in \operatorname{Sing}(\sigma)$ if and only if its corresponding $(1,0)$ or respectively $(0,1)$ maximal completion $\widehat{v} \in \operatorname{Sing}(\widehat{\sigma})$. We also show that for $\sigma \in I_{n, k}, v \in \operatorname{Sing}(\sigma)$ and $\sigma^{\prime} \in I_{n^{\prime}, k^{\prime}}$ one has $\sigma^{\prime} v_{+n^{\prime}} \in$ 
$\operatorname{Sing}\left(\sigma^{\prime} \sigma_{+n^{\prime}}\right)$ and $v \sigma_{+n}^{\prime} \in \operatorname{Sing}\left(\sigma \sigma_{+n}^{\prime}\right)$. In particular, one has $v_{+1} \in \operatorname{Sing}\left(\sigma_{+1}\right)$ (resp. $v$ as an element of $I_{n+1, k}$ is in $\operatorname{Sing}(\sigma)$ as an element of $\left.I_{n+1, k}\right)$.

Let $G r_{d}(n)$ denote $d$-Grassmanian variety of $\mathbb{C}^{n}$ that is the variety of all $d$-dimensional subspaces of $\mathbb{C}^{n}$. As a straightforward generalization of $[4, \S 6.3]$ we get

Proposition 11. Let $\sigma \in I_{n, k}$ with $2 k<n, x$ with $\lambda(x)=\left(2^{k}, 1^{n-2 k}\right)$, and $\sigma(m, n+1) \in I_{n+1, k+1}$ be its maximal $(0,1)$ completion. Then a map

$$
\phi: \mathcal{F}_{\widehat{\sigma}} \rightarrow G r_{n}(n+1): \phi\left(\left(V_{0}, \ldots V_{n+1}\right)\right)=V_{n}
$$

is the subvariety of $\mathcal{H}_{x}:=\left\{V \in G r_{n}(n+1): V \supset \operatorname{Ker} x\right\} \cong \mathbb{P}^{k}$. Moreover, the map $\phi: \mathcal{F}_{\widehat{\sigma}} \rightarrow \mathcal{H}_{x}$ is a locally trivial fiber bundle with typical fiber isomorphic to $\mathcal{F}_{\sigma}$ so that $\mathcal{F}_{\widehat{\sigma}}$ is an iterated bundle of base $\left(\mathcal{F}_{\sigma}, \mathbb{P}^{k}\right)$.

In particular one has that $\mathcal{F}_{v} \subseteq \mathcal{F}_{\sigma}$ if and only if $\mathcal{F}_{\widehat{v}} \subseteq \mathcal{F}_{\widehat{\sigma}}$ and $\operatorname{codim}_{\mathcal{F}_{\sigma}} \mathcal{F}_{v}=\operatorname{codim}_{\mathcal{F}_{\widehat{\sigma}}} \mathcal{F}_{\widehat{v}}$ and $\operatorname{dim} \mathcal{T}_{F_{v}}\left(\mathcal{F}_{\sigma}\right)-\operatorname{dim} \mathcal{F}_{\sigma}=\operatorname{dim} \mathcal{T}_{F_{\widehat{v}}}\left(\mathcal{F}_{\widehat{\sigma}}\right)-\operatorname{dim} \mathcal{F}_{\widehat{\sigma}}$, so that the components of a singular locus of $\mathcal{F}_{\widehat{\sigma}}$ are obtained by maximal completion from the components of the singular locus of $\mathcal{F}_{\sigma}$ and singular locus of $\mathcal{F}_{\widehat{\sigma}}$ is an iterated bundle of base singular locus of $\mathcal{F}_{\sigma}$ and $\mathbb{P}^{k}$.

Proof. Let $\sigma \in I_{n, k}$ with $2 k<n$ be a link pattern and $\widehat{\sigma} \in I_{n+1, k+1}$ its maximal $(0,1)$ completion. Let $\mathcal{H}_{x}$ be the variety of hyperplanes $H \subset V=\mathbb{C}^{n+1}$ such that $\operatorname{ker} x \subset H$. This is a projective variety which is naturally isomorphic to the variety of hyperplanes of the space $V / \operatorname{ker} x$, hence $\mathcal{H}_{x} \cong \mathbb{P}^{k}$.

Recall that $\mathcal{F}_{\widehat{\sigma}}=\overline{\mathcal{Z}}_{\widehat{\sigma}}$. Every flag $F=\left(V_{0}, \ldots, V_{n+1}\right) \subset \mathcal{Z}_{\widehat{\sigma}}$ satisfies ker $x \subset V_{n}$. Hence, $V_{n} \in \mathcal{H}$ whenever $F \in \mathcal{Z}_{\widehat{\sigma}}$. Thus, the fact that the map

$$
\begin{aligned}
\Phi: \mathcal{F}_{\widehat{\sigma}} & \rightarrow \mathcal{H}_{x}, \\
\left(V_{0}, \ldots, V_{n+1}\right) & \mapsto V_{n}
\end{aligned}
$$

is indeed locally trivial fiber bundle of fiber isomorphic to $\mathcal{F}_{\sigma}$ follows immediately from the fact that it is locally closed subset of $\mathcal{F}_{\sigma}$ containing $\mathcal{Z}_{\sigma}$. (or cf. the proof of [3, Theorem 2.1].)

By Proposition[11] if $\pi_{s, t}(\sigma)$ is singular and $\sigma$ is the maximal $(s-1, n-t)$-completion of $\pi_{s, t}(\sigma)$ then $\sigma$ is singular. However, it is not true that if $\mathcal{F}_{\pi_{s, t}(\sigma)}$ is singular then $\mathcal{F}_{\sigma}$ must be singular, as it is shown by the small example, not connected to admissible pairs. Consider $\sigma=(1,7)(2,6)(4,5) \in I_{7,3}$ and its projection $\pi_{1,6}(\sigma)=(2,6)(4,5) \in I_{6,2}$.

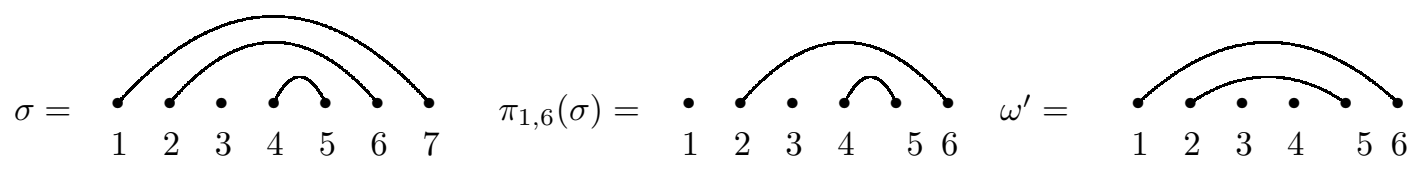

One can easily check that $\mathcal{F}_{(2,6)(4,5)}$ is singular, but $\mathcal{F}_{\sigma}$ is smooth. Indeed, $\mathcal{Z}_{\omega^{\prime}}$ is of codimension 3 in $\mathcal{F}_{\pi_{1,6}(\sigma)}$ and has 4 predecessors in $G_{\pi_{1,6}(\sigma)}$. On the other hand, by [1] if $\mathcal{F}_{\sigma}$ is singular then $\mathcal{Z}_{\omega_{0}}$ must be singular in it (where $\omega_{0}=(1,5)(2,6)(3,7)$ is from Theorem 21). However, the straightforward computation shows that there are 4 predecessors of $\omega_{0}$ in $G_{\sigma}$ and that $\operatorname{codim}_{\mathcal{F}_{\sigma}} \mathcal{Z}_{\omega_{0}}=4$, thus $\mathcal{F}_{\sigma}$ is smooth. 
For $\sigma \in I_{n, k}^{\max }$ one can use projections and concatenations in order to find elements of $\operatorname{Sing}(\sigma)$ : their number is greater or equal to the number of admissible pairs (at different intervals) of $\sigma$ obtained by repeating the procedures of adding fixed points and of maximal completions may be a few times. Note that concatenation can be represented as series of additions of fixed points and maximal completions.

Let $\mathcal{B}_{n}=\left\{e_{i}\right\}_{i=1}^{n}$ be the standard basis of $\mathbb{C}^{n}, \mathcal{B}_{n^{\prime}}^{\prime}=\left\{e_{j}^{\prime}\right\}_{j=1}^{n^{\prime}}$ be the standard basis of $\mathbb{C}^{n^{\prime}}$ and $\mathcal{B}_{n} \sqcup \mathcal{B}_{n^{\prime}}^{\prime}$ the standard basis of $\mathbb{C}^{n+n^{\prime}}$. For $x \in \operatorname{End}\left(\mathbb{C}^{n}\right), y \in \operatorname{End}\left(\mathbb{C}^{n^{\prime}}\right)$ of nilpotency order 2 of ranks $k, k^{\prime}$ respectively let $x * y \in \operatorname{End}\left(\mathbb{C}^{n+n^{\prime}}\right)$ be defined by $x * y\left(e_{i}\right)=x\left(e_{i}\right)$ for any $1 \leq i \leq n$ and $x * y\left(e_{j}^{\prime}\right)=y\left(e_{j}^{\prime}\right)$ for any $1 \leq j \leq n^{\prime}$. Obviously, $x * y$ is of nilpotency order 2 , Rank $x * y=k+k^{\prime}$ and for any $\sigma \in I_{n, k}, \sigma^{\prime} \in I_{n^{\prime}, k^{\prime}}$ one has $\mathcal{Z}_{\sigma \sigma_{+n}^{\prime}}$ is $Z_{x * y}$ orbit. We get

Proposition 12. Let $\sigma, v \in I_{n, k}$ be link patterns such that $\sigma>v$ and let $\sigma^{\prime} \in I_{n^{\prime}, k^{\prime}}$. Then $v \sigma_{+n}^{\prime} \in \operatorname{Sing}\left(\sigma \sigma_{+n}^{\prime}\right)$ iff $v \in \operatorname{Sing}(\sigma)$.

In particular, if $\widetilde{v}, \widetilde{\sigma}$ are $v, \sigma$ considered as elements of $I_{n+1, k}$ one has $\widetilde{v} \in \operatorname{Sing}(\widetilde{\sigma})$ iff $v \in \operatorname{Sing}(\sigma)$.

Proof. Indeed, note that $\operatorname{codim}_{\mathcal{F}_{\sigma \sigma_{+n}^{\prime}}} \mathcal{F}_{v \sigma_{+n}^{\prime}}=c(v)+b(v)-(c(\sigma)+b(\sigma))=\operatorname{codim}_{\mathcal{F}_{\sigma}} \mathcal{F}_{v}$.

One also has that $\sigma \sigma_{+n}^{\prime} \geq v^{\prime}>v \sigma_{+n}^{\prime}$ iff $v^{\prime}=\omega \sigma_{+n}^{\prime}$ where $\sigma \geq \omega>v$. Indeed, if $v^{\prime}=\omega \sigma_{+n}^{\prime}$ where $\sigma \geq \omega>v$ then obviously, $\sigma \sigma_{+n}^{\prime} \geq v^{\prime}>v \sigma_{+n}^{\prime}$. On the other hand, if $\sigma \sigma_{+n}^{\prime} \geq v^{\prime}>v \sigma_{+n}^{\prime}$, then in particular, $\sigma_{+n}^{\prime}=\pi_{n+1, n+n^{\prime}}\left(\sigma \sigma_{+n}^{\prime}\right) \leq \pi_{n+1, n+n^{\prime}}\left(v^{\prime}\right) \leq \pi_{n+1, n+n^{\prime}}\left(v \sigma_{+n}^{\prime}\right)=\sigma_{+n}^{\prime}$. Thus, $\pi_{n+1, n+n^{\prime}}\left(v^{\prime}\right)=\sigma_{+n}^{\prime}$. One also has $\pi_{1, n}\left(\sigma \sigma_{+n}^{\prime}\right)=\sigma \geq \pi_{1, n}\left(v^{\prime}\right) \geq \pi_{1, n}\left(v \sigma_{+n}^{\prime}\right)=v$. Taking into account that the number of arcs in all link patterns are $k+k^{\prime}$ we get that $v^{\prime}=\omega \sigma_{+n}^{\prime}$ where $\omega=\pi_{1, n}\left(v^{\prime}\right)$ satisfies $\sigma \geq \omega>v$.

In particular $v^{\prime} \leq \sigma \sigma_{+n}^{\prime}$ is a predecessor of $v \sigma_{+n}^{\prime}$ iff $\omega \leq \sigma$ is a predecessor of $v$. Thus,

$$
\operatorname{dim} \mathcal{T}_{F_{v \sigma_{+n}^{\prime}}}\left(\mathcal{F}_{\sigma \sigma_{+n}^{\prime}}\right)-\operatorname{dim} \mathcal{F}_{\sigma \sigma_{+n}^{\prime}}=\operatorname{dim} \mathcal{T}_{F_{v}}\left(\mathcal{F}_{\sigma}\right)-\operatorname{dim} \mathcal{F}_{\sigma}
$$

\subsection{The components of the singular locus of a $\mathcal{F}_{\sigma}$ for $\sigma \in I_{n, k}^{\max }$}

The algorithm for elements of $\operatorname{Sing}(\sigma)$ for $\sigma \in I_{n, k}^{\max }$ with $\rho(\sigma) \geq 4$ is obtained as follows:

a) completion - determining $\widehat{\sigma}$ :

- If $1, n \in \sigma^{0}$ put $\widehat{\sigma}=\sigma$ and $s=1, t=n$;

- If $\left|\{1, n\} \cap \sigma^{0}\right|=1$ then if $1 \in \sigma^{0}$ put $s=1, t=\max \tau^{*}(\sigma)$ and $\widehat{\sigma}=\pi_{1, t}(\sigma)$, otherwise put $t=n, s=\min \tau^{*}(\sigma)+1$ and $\widehat{\sigma}=\left(\pi_{s, n}(\sigma)\right)_{+(1-s)}$;

- If $\{1, n\} \cap \sigma^{0}=\emptyset$ and $(1, n) \in \sigma$ put $s=1, t=\max \tau^{*}(\sigma)$ and $\widehat{\sigma}=\pi_{1, t}(\sigma)$; otherwise put $s=\min \tau^{*}(\sigma)+1, t=\max \tau^{*}(\sigma)$ and $\widehat{\sigma}=\left(\pi_{s, t}(\sigma)\right)_{+(1-s)}$.

In step (b) we find $\operatorname{Sing}(\widehat{\sigma})$ and then get $\operatorname{Sing}(\sigma)$ from it by maximal $(s-1, n-t)$-completion. 
b) For any $\{i, j\} \in \tau^{*}(\widehat{\sigma})$ let $(a, b) \in \widehat{\sigma}$ be maximal arc over $(i, i+1)$ such that $b<j$ and $\tau^{*}(\widehat{\sigma}) \cap[a, i-1]=\emptyset$. Let $(c, d)$ be maximal arc over $(j, j+1)$ such that $i<c$ and $\tau^{*}(\widehat{\sigma}) \cap$ $[j+1, d]=\emptyset$. Note that these arcs are well defined and $b<c$ since $\widehat{\sigma}$ is maximal. Now for $(a, b),(c, d)$ find all possible intervals, where they are an admissible pair. To do this, put $m_{1}=\max \left\{f \in \widehat{\sigma}^{0}: f<a\right\}$ and $m_{2}=\min \left\{f \in \widehat{\sigma}^{0}: f>d\right\}$ and put $v_{1}=\pi_{1, m_{1}-1}(\widehat{\sigma})$, $v_{2}=\pi_{m_{2}+1, n}(\widehat{\sigma})$ and $v=\pi_{m_{1}, m_{2}}(\widehat{\sigma})$.

- If $\tau^{*}(\widehat{\sigma}) \cap\left(\left[m_{1}, a\right] \cup\left[d, m_{2}\right]\right)=\emptyset$ then $\left[m_{1}, m_{2}\right]$ is the only possible interval for $(a, b),(c, d)$ to be admissible at and $\omega=v_{1}\left(v_{(a, b)(c, d)}^{-}(a, d)\left(m_{1}, m_{2}\right)\right) v_{2}$.

- Otherwise,

If there is no $(s, t) \in v$ such that $s<a<b<t<j$ then for $l \in \tau^{*}(\widehat{\sigma}) \cap\left(\left[m_{1}, a\right]\right)$ let $\left(r_{l}, q_{l}\right)$ be maximal arc of $v$ over $(l, l+1)$ such that $q_{l}<a$ and let $I=\left\{m_{1}\right\} \cup\left\{q_{l}\right\}_{l \in \tau^{*}(\widehat{\sigma}) \cap\left(\left[m_{1}, a\right]\right)}$. If there exists $(s, t) \in v$ such that $s<a<b<t<j$ then let $(s, t)$ be minimal such arc. Note that $\tau^{*}(\widehat{\sigma}) \cap[s, a] \neq \emptyset$ and for $l \in \tau^{*}(\widehat{\sigma}) \cap[s, a]$ let $\left(r_{l}, q_{l}\right)$ be maximal arc of $v$ over $(l, l+1)$ such that $q_{l}<a$ and let $I=\left\{q_{l}\right\}_{l \in \tau^{*}(\widehat{\sigma}) \cap[s, a]}$.

Exactly in the same way,

If there is no $(s, t) \in v$ such that $i<s<c<d<t$ then for $l \in \tau^{*}(\widehat{\sigma}) \cap\left(\left[d, m_{2}\right]\right)$ let $\left(r_{l}, q_{l}\right)$ be maximal arc of $v$ over $(l, l+1)$ such that $d<r_{l}$ and let $J=\left\{m_{2}\right\} \cup\left\{r_{l}\right\}_{l \in \tau^{*}(\widehat{\sigma}) \cap\left(\left[d, m_{2}\right]\right)}$. If there exists $(s, t) \in v$ such that $i<s<c<d<t$ then let $(s, t)$ be minimal such arc. Note that $\tau^{*}(\widehat{\sigma}) \cap[d, t] \neq \emptyset$ and for $l \in \tau^{*}(\widehat{\sigma}) \cap[d, t]$ let $\left(r_{l}, q_{l}\right)$ be maximal arc of $v$ over $(l, l+1)$ such that $r_{l}>d$ and let $J=\left\{r_{l}\right\}_{l \in \tau^{*}(\widehat{\sigma}) \cap[d, t]}$.

For any interval $[p, q]: r \in I, q \in J$ the pair $(a, b),(c, d)$ is admissible at $[r, q]$ and $\omega([r, q])=v_{1} \widehat{\omega} v_{2}$ where

if $r>m_{1}$ and $q<m_{2}$ then $\widehat{\omega}$ is $\left(r-2-m_{1}, m_{2}-q-1\right)$-completion of

$$
\pi_{r, q}(v)_{(a, b),(c, d)}^{-}(a, d)(r, q) .
$$

This completion belongs to $\left[m_{1}+1, m_{2}-1\right]$ so that $m_{1}, m_{2}$ are fixed points; if $r=m_{1}$ and $q<m_{2}$ then $\widehat{\omega}$ is $\left(0, m_{2}-q-1\right)$-completion of $\pi_{m_{1}, q}(v)_{(a, b),(c, d)}^{-}(a, d)\left(m_{1}, q\right)$ with one added fixed point on the right; if $r>m_{1}$ and $q=m_{2}$ then $\widehat{\omega}$ is $\left(r-2-m_{1}, 0\right)$-completion of $\pi_{r, m_{2}}(v)_{(a, b),(c, d)}^{-}(a, d)\left(r, m_{2}\right)$ with one added fixed point on the left; Finally, if $(r, q)=\left(m_{1}, m_{2}\right)$ then $\widehat{\omega}=v_{(a, b)(c, d)}^{-}(a, d)\left(m_{1}, m_{2}\right)$.

Example 13. Consider the following example, demonstrating different cases of our algorithm. Let $\sigma=(2,9)(3,6)(4,5)(7,8)(10,11) \in I_{12,5}$

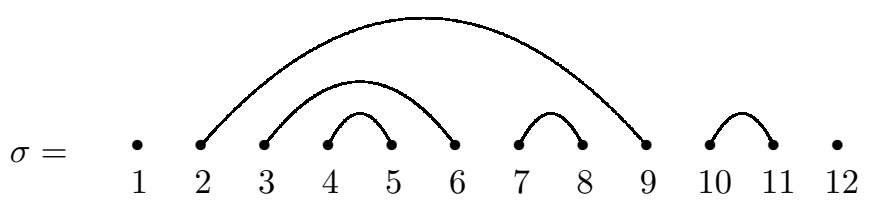

$\tau^{*}(\sigma)=\{4,7,10\}$. For $\{4,7\}$ the admissible pair is $(3,6),(7,8)$ and the intervals are $[1,10]$ and $[1,12]$ with respective $\omega_{1}, \omega_{2} \in \operatorname{Sing}(\sigma)$ : 

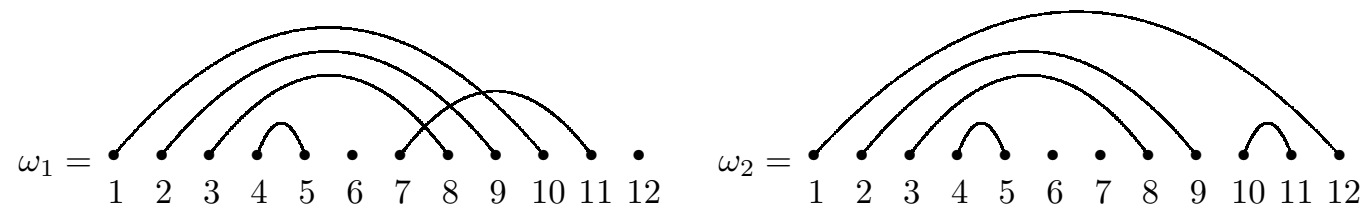

For $\{4,10\}$ the admissible pair is $(2,9),(10,11)$ and the only interval is $[1,12]$. Respectively,

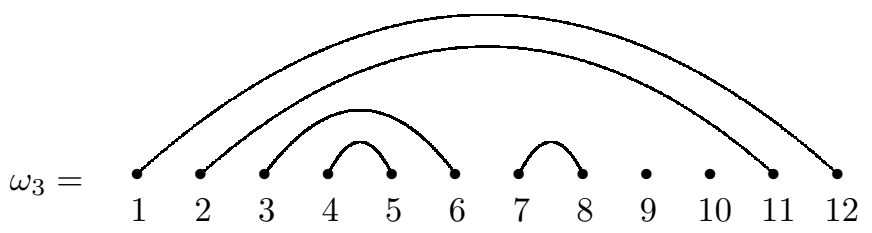

Finally, for $\{7,10\}$ the admissible pair is $(7,8),(10,11)$ and the only interval $[6,12]$ so that

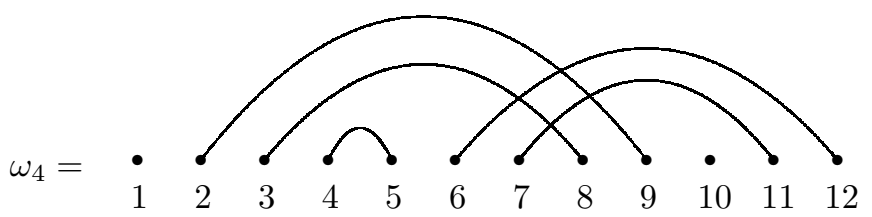

So, according to the algorithm there are 4 elements in $\operatorname{Sing}(\sigma)$.

We are going to show in this subsection that for $\sigma \in I_{n, k}$ with $\rho(\sigma) \geq 4, \operatorname{Sing}(\sigma)$ is obtained by this algorithm.

Theorem 14. Let $\sigma \in I_{n, k}^{\max }$ be such that $\rho(\sigma) \geq 4$ and let $\omega \in \operatorname{Sing}(\sigma)$ then $\omega$ is obtained by our algorithm.

To prove the theorem we need a few technical lemmas. Then we prove it in Propositions 21, 22, Recall a notion of a predecessor of $\sigma \in I_{n, k}$ from $\S 2.2$.

Lemma 15. Given $\sigma \in I_{n, k}^{\max }$ with $b(\sigma)+c(\sigma)=k$ then the number of predecessors of $\sigma$ is $2 k$.

Proof. Indeed for each fixed point $f$ and $(i, j) \in \sigma$ a bridge over it one has $\sigma^{\prime}=(i, f) \sigma_{(i, j)}^{-}$and $\sigma^{\prime \prime}=(f, j) \sigma_{(i, j)}^{-}$are predecessors connected to this point and this bridge.

For $(i, j)(s, t) \in \sigma$ such that $i<s<j<t$ one has

$$
\sigma^{\prime}=(i, s)(t, j) \sigma_{(i, j),(s, t)}^{-} \text {and } \sigma^{\prime \prime}=(i, t)(s, j) \sigma_{(i, j),(s, t)}^{-}
$$

are predecessors connected to these two crossing arcs.

Thus we get that each crossing and each bridge over a fixed point provides us exactly 2 predecessors. 
Recall that for $\sigma, \omega \in I_{n, k}$ such that $\sigma>\omega$ and for their $(0,1)$-maximal completions $\sigma(a, n+$ $1), \omega(b, n+1)$ one has $\sigma(a, n+1)>\omega(b, n+1)$ by Proposition 11, Note also that the elementary moves on $\sigma$ and its maximal completion correspond, namely

Lemma 16. Let $\sigma \in I_{n, k}$ and let $f=\max \sigma^{0}$. Let $\widehat{\sigma}=\sigma(f, n+1)$ be its $(0,1)$-maximal completion. Then for any predecessor $\sigma^{\prime}$ of $\sigma$ there exists $\widehat{\sigma}^{\prime}$ predecessor of $\widehat{\sigma}$ obtained by completion (not necessarily maximal).

Proof. If $\sigma^{\prime}$ is a predecessor of $\sigma$ obtained by any elementary move forward, not connected to $f$ then respectively $\sigma^{\prime}(f, n+1)$ is the corresponding predecessor of $\widehat{\sigma}$ (note that it must not be the maximal completion of $\left.\sigma^{\prime}\right)$.

If there exist $(a, b) \in \sigma$ such that $a<f<b$ then for predecessor $\sigma^{\prime}=\sigma_{(a, b)}^{-}(a, f)$ one has $\widehat{\sigma}^{\prime}=\sigma_{(a, b)}^{-}(a, f)(b, n+1)$ is the corresponding predecessor of $\widehat{\sigma}$ and for predecessor $\sigma^{\prime \prime}=\sigma_{(a, b)}^{-}(f, b)$ one has $\widehat{\sigma}^{\prime \prime}=\sigma_{(a, b)}^{-}(a, n+1)(f, b)$ is the corresponding predecessor of $\widehat{\sigma}$ (again in this case $\widehat{\sigma}^{\prime \prime}$ is not necessarily maximal completion of $\left.\sigma^{\prime \prime}\right)$.

As a corollary we get:

Proposition 17. Given $\sigma \in I_{n, k}^{\max }$ and $\omega \in G_{\sigma} \backslash\{\sigma\}$ then for every pair $\left\{\omega^{\prime}, \omega^{\prime \prime}\right\}$ of predecessors of $\omega$ at least one is in $G_{\sigma}$.

Proof. This claim is true only for $\sigma \in I_{n, k}^{\max }$, so its proof is rather long and technical. We simply make all possible cases one by one. For $\sigma \in I_{n, k}^{\max }, \omega \in G_{\sigma}$ and its predecessor $\omega^{\prime}$ satisfying $\omega^{\prime} \leq \sigma$ one has by Proposition 11 and Lemma 16 that for $(0,1)$-maximal completions $\widehat{\sigma}, \widehat{\omega}$ there exists completion $\widehat{\omega}^{\prime}$ satisfying $\widehat{\omega}^{\prime}$ is a predecessor of $\widehat{\omega}$ and $\widehat{\omega}^{\prime} \leq \widehat{\sigma}$. Thus, using these two claims $n-2 k$ times it is enough to show the claim only for $\sigma, \omega \in I_{2 k, k}$, that is $k$-complete link patterns where all the predecessors are obtained by "uncrossing" a given cross. We will show the claim by induction on the number of crosses in $\omega$.

If $c(\omega)=1$ then obviously $\sigma$ is one of the only two predecessors of $\omega$ so the claim is trivially true.

Now assume that this is true for $\omega^{\prime}$ with at most $j-1$ crosses and show for $\omega$ with $j$ crosses.

By the construction of $G_{\sigma}$ there exists $v \in G_{\sigma}$ such that $\omega=(a, c)(b, d) \phi$ where $a<b<c<d$ and $v=(a, b)(c, d) \phi$ or $v=(a, d)(b, c) \phi$ and in both cases $c r(v)=j-1$. Before we proceed, note that since $\operatorname{codim}_{\mathcal{F}_{v}} \mathcal{Z}_{\omega}=1$ :

(1) if $v=(a, b)(c, d) \phi$ then there are no $(i, j) \in \phi$ such that $i<a, b<j<c$ or $b<i<c, d<j$;

(2) if $v=(a, d)(b, c) \phi$ then there are no $(i, j) \in \phi$ such that $a<i<b$ and $c<j<d$.

There are 3 categories of predecessors of $\omega$ :

(i) connected to $(p, r)(q, s) \in \phi$ such that $p<q<r<s$;

(ii) connected either to $(a, c)$ and $(i, j) \in \phi$ crossing $(a, c)$ that is such that either $a<i<c<j$ or $i<a<j<c$; or respectively connected to $(b, d)$ and $(i, j) \in \phi$ crossing $(b, d)$ that is such that either $i<b<j<d$ or $b<i<d<j$. 
(iii) $(a, b)(c, d) \phi$ and $(a, d)(b, c) \phi$.

By our construction at least one of predecessors in (iii) satisfies the claim. Let us study the predecessors of types (i) and (ii)

(i) For any $(p, r)(q, s) \in \phi$ such that $p<q<r<s$ and for $v=(a, b)(c, d) \phi$ or $(a, d)(b, c) \phi$ one has by induction assumption that either predecessor $v^{\prime}=v_{(p, r)(q, s)}^{-}(p, q)(r, s)<\sigma$ or or predecessor $v^{\prime}=v_{(p, r)(q, s)}^{-}(p, s)(q, r)<\sigma$. In both cases

$$
\omega^{\prime}= \begin{cases}\left(v^{\prime}\right)_{(a, b)(c, d)}^{-}(a, c)(b, d) & \text { if } v=(a, b)(c, d) \phi ; \\ \left(v^{\prime}\right)_{(a, d)(b, c)}^{-}(a, c)(b, d) & \text { if } v=(a, d)(b, c) \phi ;\end{cases}
$$

is a predecessor of $\omega$ satisfying $\omega^{\prime}<v^{\prime}$ so that $\omega^{\prime}<\sigma$ and we are done.

(ii) Obviously, case $(i, j)$ intersecting $(a, c)$ and case $(i, j)$ intersecting $(b, d)$ are obtained one from another by Schützenberger transformation of $\sigma, \omega$, so it is enough to consider only the case $(i, j)$ intersecting $(a, c)$. Here we consider two types of $v$ separately.

First assume $v=(a, b)(c, d) \phi$.

- Let $(i, j) \in \phi$ be such that $i<a<j<c$. The corresponding predecessors of $\omega$ are $\omega^{\prime}=(i, a)(j, c)(b, d) \phi_{(i, j)}^{-}$and $\omega^{\prime \prime}=(i, c)(a, j)(b, d) \phi_{(i, j)}^{-}$. By $(1)$ one has $i<a<j<b$ so that $v$ has two predecessors $v^{\prime}=(i, a)(j, b)(c, d) \phi_{(i, j)}^{-}$and $v^{\prime \prime}=(i, b)(a, j)(c, d) \phi_{(i, j)}^{-}$connected to crossing $\operatorname{arcs}(i, j),(a, b)$. By induction hypothesis either $v^{\prime} \leq \sigma$ or $v^{\prime \prime} \leq \sigma$. Note that $v^{\prime}$ is a predecessor of $\omega^{\prime}$ and $v^{\prime \prime}$ is a predecessor of $\omega^{\prime \prime}$ so that at least one of $\omega^{\prime}, \omega^{\prime \prime}$ is smaller than $\sigma$.

- Let $(i, j) \in \phi$ be such that $a<i<c<j$. The corresponding predecessors of $\omega$ are $\omega^{\prime}=$ $(a, i)(c, j)(b, d) \phi_{(i, j)}^{-}$and $\omega^{\prime \prime}=(a, j)(i, c)(b, d) \phi_{(i, j)}^{-}$. By (1) one has either $b<i<c<j<d$ or $i<b$.

If $b<i<c<j<d$ then

$$
\begin{aligned}
v^{\prime} & =(a, b)(i, d)(c, j) \phi_{(i, j)}^{-}, \\
v^{\prime \prime} & =(a, b)(i, c)(j, d) \phi_{(i, j)}^{-}
\end{aligned}
$$

and are predecessors of $v$ connected to $\operatorname{arcs}(i, j)$ and $(c, d)$. By induction hypothesis at least one of them is in $G_{\sigma}$. Further note that $v^{\prime}$ is a predecessor of $\omega^{\prime}$ and $v^{\prime \prime}$ is a predecessor of $\omega^{\prime \prime}$ so that at least one of $\omega^{\prime}, \omega^{\prime \prime}$ is smaller than $\sigma$.

If $i<b$ then $v^{\prime}=(a, i)(b, j)(c, d) \phi_{(i, j)}^{-}$and $v^{\prime \prime}=(a, j)(i, b)(c, d) \phi_{(i, j)}^{-}$are the predecessors of $v$ connected to arcs $(a, b),(i, j)$ and by induction hypothesis at least one of them is less or equal to $\sigma$.

- If in addition $j>d$ then $v^{\prime}$ is a predecessor of $\omega^{\prime}$ and $v^{\prime \prime}$ is a predecessor of $\omega^{\prime \prime}$ so that at least one of $\omega^{\prime}, \omega^{\prime \prime}$ is less than $\sigma$.

- The case $j<d$ is more subtle and we draw the corresponding link patterns in order to see the picture. Since all the arcs but connected to $a, b, c, d, i, j$ are the same in all our link patterns we can ignore them and draw only those connected to these 6 points. One has: 

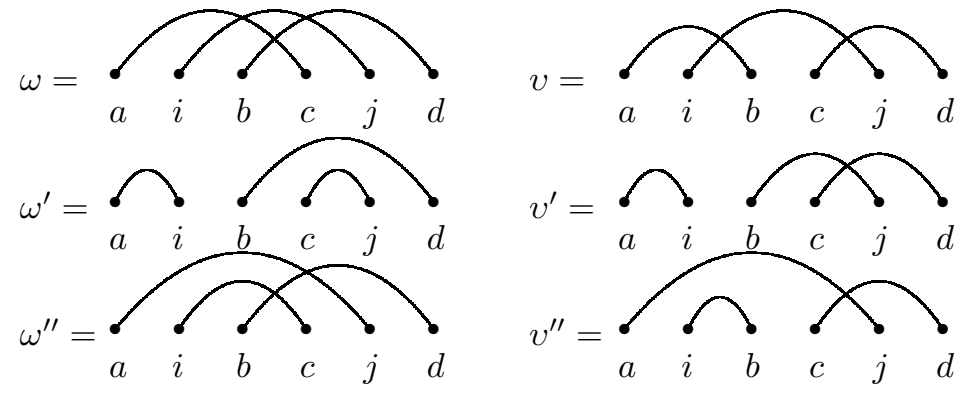

If $v^{\prime \prime}<\sigma$ then since $v^{\prime \prime}$ is a predecessor of $\omega^{\prime \prime}$ we are done. If $v^{\prime}<\sigma$ then consider its predecessors connected to $\operatorname{arcs}(b, j),(c, d)$. They are $v_{1}^{\prime}=(a, i)(b, j)(c, d) \phi_{(i, j)}^{-}$and $v_{2}^{\prime}=(a, i)(b, c)(j, d) \phi_{(i, j)}^{-}$. By induction hypothesis applied to $v^{\prime}$ either $v_{1}^{\prime} \leq \sigma$ or $v_{2}^{\prime} \leq \sigma$. Note that $v_{1}^{\prime}=\omega^{\prime}$ thus in this case we are done. If $v_{2}^{\prime} \leq \sigma$ note that $v_{2}^{\prime}$ is a predecessor of $(a, j)(i, d)(b, c) \phi_{(i, j)}^{-}$which is in turn is a predecessor of $\omega^{\prime \prime}$ so that in this case again $\omega^{\prime \prime}<\sigma$. Thus, in this case also at least one of $\omega^{\prime}, \omega^{\prime \prime}$ less than $\sigma$.

Consider case $v=(a, d)(b, c) \phi$.

- Let $(i, j) \in \phi$ be such that $i<a<j<c$. The corresponding predecessors of $\omega$ are $\omega^{\prime}=(i, a)(j, c)(b, d) \phi_{(i, j)}^{-}$and $\omega^{\prime \prime}=(i, c)(a, j)(b, d) \phi_{(i, j)}^{-}$.

- If in addition $j<b$ then $(i, j)$ crosses $(a, d)$ in $v$ and does not cross $(b, c)$. The predecessors of $v$ are $v^{\prime}=(i, a)(j, d)(b, c) \phi_{(i, j)}^{-}$and $v^{\prime \prime}=(i, d)(a, j)(b, c) \phi_{(i, j)}^{-}$. By induction hypothesis at least one of them is less or equal to $\sigma$ and as one can see immediately $v^{\prime}$ is a predecessor of $\omega^{\prime}$ and $v^{\prime \prime}$ is a predecessor of $\omega^{\prime \prime}$ so that at least one of $\omega^{\prime}, \omega^{\prime \prime}$ less than $\sigma$.

- The case $j>b$ is more subtle and and we draw the corresponding link patterns in order to see the picture. We again draw only arcs connected to $a, b, c, d, i, j$. One has:
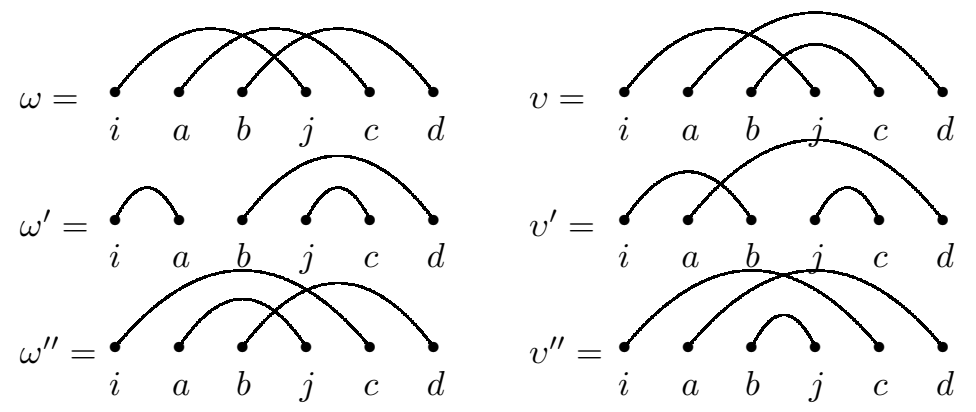

and by induction hypothesis $v^{\prime}<\sigma$ or $v^{\prime \prime}<\sigma$. Note that $v^{\prime \prime}$ is a predecessor of $\omega^{\prime \prime}$ so that if $v^{\prime \prime}<\sigma$ we are done. If $v^{\prime}<\sigma$ then by induction hypothesis at least one 
of predecessors connected to $\operatorname{arcs}(i, b),(a, d)$ is less or equal to $\sigma$. These predecessors are $v_{1}^{\prime}=(i, a)(b, d)(j, c) \phi_{(i, j)}^{-}=\omega^{\prime}$ and $v_{2}^{\prime}=(i, d)(a, b)(j, c) \phi_{(i, j)}^{-}$. One has $v_{2}^{\prime}$ is a predecessor of $(i, c)(a, b)(j, d) \phi_{(i, j)}^{-}$which is a predecessor of $\omega^{\prime \prime}$, so that in this case also either $\omega^{\prime}<\sigma$ or $\omega^{\prime \prime}<\sigma$.

- Let $(i, j) \in \phi$ be such that $a<i<c<j$. The corresponding predecessors of $\omega$ are $\omega^{\prime}=(a, i)(b, d)(c, j) \phi_{(i, j)}^{-}$and $\omega^{\prime \prime}=(a, j)(i, c)(b, d) \phi_{(i, j)}^{-}$. By $(2)$ one has either $i>b$ or $i<b$ and $j>d$.

If $i<b$ and $j>d$ then $v=(a, d)(i, j)(b, c) \phi_{(i, j)}^{-}$has predecessors $v^{\prime}=(a, i)(b, c)(d, j) \phi_{(i, j)}^{-}$ and $v^{\prime \prime}=(a, j)(i, d)(b, c) \phi_{(i, j)}^{-}$connected to arcs $(a, d),(i, j)$. By induction hypothesis at least one of them is less or equal to $\sigma$ and since $v^{\prime}$ is a predecessor of $\omega^{\prime}$ and $v^{\prime \prime}$ is a predecessor of $\omega^{\prime \prime}$ we are done.

If $i>b$ and $j<d$ then $v=(a, d)(b, c)(i, j) \phi_{(i, j)}^{-}$has predecessors $v^{\prime}=(a, d)(b, i)(c, j) \phi_{(i, j)}^{-}$ and $v^{\prime \prime}=(a, d)(b, j)(i, c) \phi_{(i, j)}^{-}$connected to arcs $(b, c),(i, j)$ and since $v^{\prime}$ is a predecessor of $\omega^{\prime}$ and $v^{\prime \prime}$ is a predecessor of $\omega^{\prime \prime}$ by induction hypothesis applied to $v$ we are done.

Finally, the case $i>b$ and $j>d$ is more subtle so we again use a picture. One has:
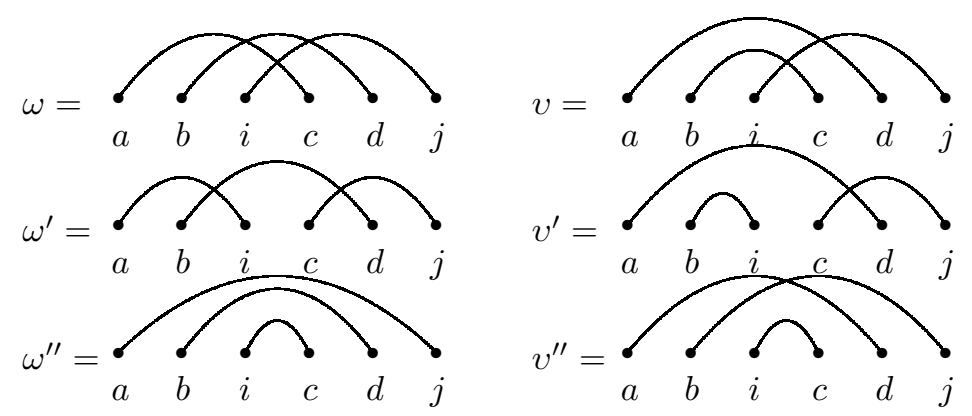

If $v^{\prime}<\sigma$ then since it is a predecessor of $\omega^{\prime}$ we get $\omega^{\prime}<\sigma$. If $v^{\prime \prime}<\sigma$ then one of its predecessors $v_{1}^{\prime \prime}=(a, j)(b, d)(i, c) \phi_{(i, j)}^{-}=\omega^{\prime \prime}$ or $v_{2}^{\prime \prime}=(a, b)(i, c)(d, j) \phi_{(i, j)}^{-}$connected to arcs $(a, d),(b, j)$ is less or equal to $\sigma$. Thus it is enough to note that $v_{2}^{\prime \prime}$ is a predecessor of $(a, i)(b, c)(d, j) \phi_{(i, j)}^{-}$which is in turn is a predecessor of $\omega^{\prime}$, thus again at least one of $\omega^{\prime}, \omega^{\prime \prime}$ is less or equal to $\sigma$.

As a straightforward corollary we get

Corollary 18. Given $\sigma \in I_{n, k}^{\max }$ and $\omega \in G_{\sigma} \backslash\{\sigma\}$, one has $\mathcal{F}_{\omega}$ is in the singular locus of $\mathcal{F}_{\sigma}$ if and only if either there exists $(a, b) \in \omega$ and fixed point $i: a<i<b$ such that both $\omega_{(a, b)}^{-}(a, i), \omega_{(a, b)}^{-}(i, b)<\sigma$ or there exists crossing pair $(a, b),(c, d) \in \omega$ where $a<c<b<d$ such that both $\omega_{(a, b)(c, d)}^{-}(a, c)(b, d), \omega_{(a, b)(c, d)}^{-}(a, d)(c, b)<\sigma$. 
For $\sigma=\left(i_{1}, j_{1}\right) \ldots\left(i_{k}, j_{k}\right) \in I_{n, k}$ put

$$
\widetilde{\sigma}^{\left(i_{s}, j_{s}\right)}=\left(i_{1}^{\prime}, j_{1}^{\prime}\right) \ldots\left(i_{s-1}^{\prime}, j_{s-1}^{\prime}\right)\left(i_{s+1}^{\prime}, j_{s+1}^{\prime}\right) \ldots\left(i_{k}^{\prime}, j_{k}^{\prime}\right)
$$

to be a link pattern in $I_{n-1, k-1}$ obtained by

$$
d_{t}^{\prime}= \begin{cases}d_{t} & \text { if } d_{t}<i_{s} \\ d_{t}-1 & \text { if } i_{s}<d_{t}<j_{s} \\ d_{t}-2 & \text { if } d_{t}>j_{s}\end{cases}
$$

for any $d_{t} \in\left\{i_{r}, j_{r}\right\}_{r=1, r \neq s}^{k}$. In other words we extract $\left(i_{s}, j_{s}\right)$ together with the points $i_{s}, j_{s}$.

Lemma 19. Let $\sigma \in I_{n, k}^{\max }$ and $v \in I_{n, k}$ be such that $(i, i+1) \in \sigma, v$. Then $\widetilde{v}^{(i, i+1)} \in G_{\widetilde{\sigma}^{(i, i+1)}}$ and $F_{\widetilde{v}(i, i+1)}$ is a singular point of $\mathcal{F}_{\widetilde{\sigma}^{(i, i+1)}}$ if and only if $v \in G_{\sigma}$ and respectively $F_{v}$ is a singular point of $\mathcal{F}_{\sigma}$. In particular, $v \in \operatorname{Sing}(\sigma)$ iff $\widetilde{v}^{(i, i+1)} \in \operatorname{Sing}\left(\widetilde{\sigma}^{(i, i+1)}\right)$.

Proof. Both parts of the Lemma are immediate. For the first part it is enough to note that for $\omega$ such that $(i, i+1) \in \omega$ one has

$$
R_{s, t}(\omega)= \begin{cases}R_{s, t}\left(\widetilde{\omega}^{(i, i+1)}\right) & \text { if } t \leq i ; \\ R_{s-2, t-2}\left(\widetilde{\omega}^{(i, i+1)}\right) & \text { if } s \geq i+1 ; \\ R_{s, t-2}\left(\widetilde{\omega}^{(i, i+1)}\right)+1 & \text { if } s<i+1, t>i ;\end{cases}
$$

Thus $R_{s, t}(\sigma) \geq R_{s, t}(v)$ for all $1 \leq s<t \leq n$ if and only if $R_{s, t}\left(\widetilde{\sigma}^{(i, i+1)}\right) \geq R_{s, t}\left(\widetilde{v}^{(i, i+1)}\right)$ for any $1 \leq s<t \leq n-2$. Moreover since for any predecessor $v^{\prime}$ of $v$ one has $(i, i+1) \in v^{\prime}$ we get exactly the same result for $v^{\prime}$.

In our next lemma we concentrate on $\omega \in \operatorname{Sing}(\sigma)$.

Lemma 20. Let $\sigma \in I_{n, k}^{\max }$ with $\rho(\sigma) \geq 4$ and let $\omega \in \operatorname{Sing}(\sigma)$ such that there exists $f \in \omega^{0}$ and $(i, j) \in \omega$ such that $i<f<j$ and both $\omega_{(i, j)}^{-}(i, f), \omega_{(i, j)}^{-}(f, j) \in G_{\sigma}$. Let $(a, b) \in \omega$ be minimal such arc, that is for any $(i, j) \in \omega$ satisfying the condition, one has $(i, j) \notin[a, b]$. Put $k_{1}=|\{(i, j) \in \sigma: i<f<j\}|$. Let $S=\{(i, j) \in \omega: i<f<j\}$. One has

(i) $|S| \geq k_{1}+2$;

(ii) All arcs of $S$ are concentric (that is for any $(i, j),\left(i^{\prime}, j^{\prime}\right) \in S$ such that $i<i^{\prime}$ one has $i<i^{\prime}<$ $j^{\prime}<j$ and $(a, b)$ is the minimal arc of $S$, that is if $(i, j) \in S \backslash\{(a, b)\}$ then $i<a<b<j$. For any $(i, j) \in S$ and any $f^{\prime} \in \omega^{0} \cap[a, b]$ one has $\omega_{(i, j)}^{-}\left(i, f^{\prime}\right), \omega_{(i, j)}^{-}\left(f^{\prime}, j\right) \in G_{\sigma}$, and there are no fixed points at $[i, j] \backslash[a, b]$ for any $(i, j) \in S$.

(iii) For any $(i, j) \in \omega \backslash S$ there are no fixed points under $(i, j)$ and any $(i, j),\left(i^{\prime}, j^{\prime}\right)$ in $\omega \backslash S$ do not intersect, and if $(i, j) \in \omega \backslash S$ intersects any $(c, d) \in S$ then $(i, j)$ intersects all the arcs of $S$.

Proof. (i) Let $k_{2}=|\{(i, j) \in \sigma: j \leq f\}|$ and $k_{3}=|\{(i, j) \in \sigma: i \geq f\}|$ then $k=k_{1}+k_{2}+k_{3}$. Exactly in the same manner for $v \in G_{\sigma}$ let $m_{1}(v)=|\{(i, j) \in v: i<f<j\}|, m_{2}(v)=\mid\{(i, j) \in$ $\omega: j \leq f\} \mid$ and $m_{3}(v)=|\{(i, j) \in v: i \geq f\}|$. Again one has $m_{1}(v)+m_{2}(v)+m_{3}(v)=k$ and 
since $v \leq \sigma$ we get $m_{2}(v) \leq k_{2}$ and $m_{3}(v) \leq k_{3}$. One also has $m_{2}\left(\omega_{(a, b)}^{-}(a, f)\right)=m_{2}(\omega)+1 \leq k_{2}$ and $m_{3}\left(\omega_{(a, b}^{-}(f, b)=m_{3}(\omega)+1 \leq k_{3}\right.$. Thus $|S|=k-m_{2}(\omega)-m_{3}(\omega) \geq k_{1}+2$.

(ii) We have to consider the following three cases:

(a) There exists $(c, d) \in \omega$ such that $a<c<f<d<b$. We show in this case that there exists $\omega^{\prime}>\omega$ such that $\omega^{\prime}$ is singular in $G_{\sigma}$.

(b) There exists $(c, d) \in S$ crossing $(a, b)$. Again, we show in this case that there exists $\omega^{\prime}>\omega$ such that $\omega^{\prime}$ is singular in $G_{\sigma}$.

(c) There exists $(c, d) \in S$ and exists $p \in \omega^{0} \backslash[a, b]$ with $c<p<d$. Again, we show in this case that there exists $\omega^{\prime}>\omega$ such that $\omega^{\prime}$ is singular in $G_{\sigma}$.

Since all three cases are shown in the same way, we will consider in detail only case (a). Assume first that there exists $(c, d) \in \omega$ such that $a<c<f<d<b$. By Proposition 17 and minimality of $(a, b)$ one has that exactly one out of $\omega_{(c, d)}^{-}(c, f), \omega_{(c, d)}^{-}(f, d)$ is in $G_{\sigma}$. Without loss of generality we can assume that this is $v=\omega_{(c, d)}^{-}(c, f)$. One has

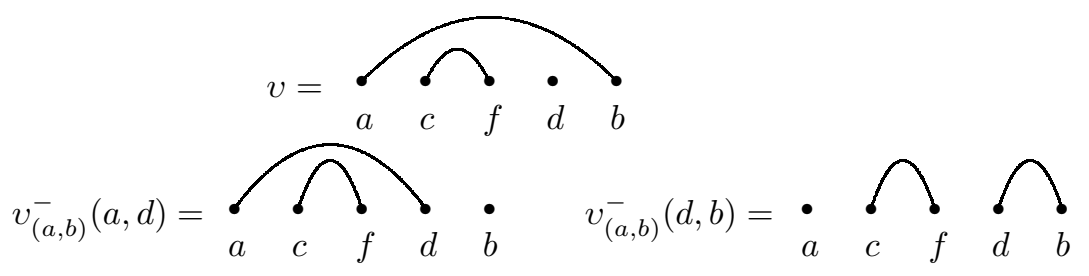

Again, one of $v_{(a, b)}^{-}(a, d), v_{(a, b)}^{-}(d, b)$ is in $G_{\sigma}$.

- If $v_{(a, b)}^{-}(a, d) \in G_{\sigma}$ recall that $\omega^{\prime}=\omega_{(a, b)}^{-}(f, b)<\sigma$ so that

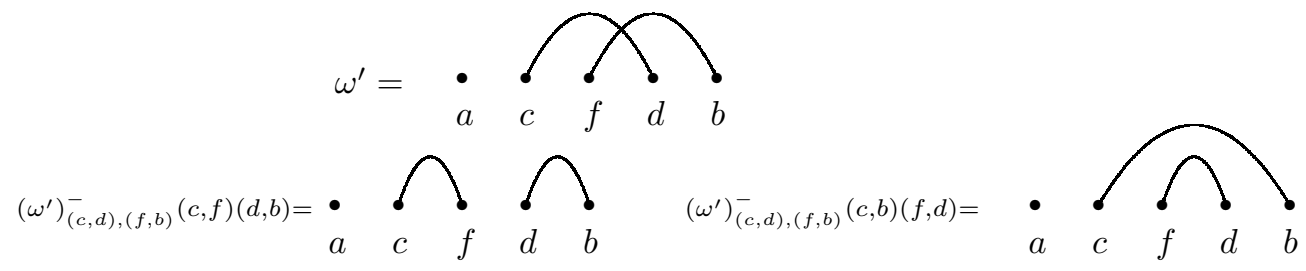

If $\left(\omega^{\prime}\right)_{(c, d),(f, b)}^{-}(c, f)(d, b)<\sigma$ then we get that $v$ satisfies $v>\omega$ and both predecessors $v_{(a, b)}^{-}(a, d), v_{(a, b)}^{-}(d, b)<\sigma$ so that $\omega \notin \operatorname{Sing}(\sigma)$.

If $\left(\omega^{\prime}\right)_{(c, d),(f, b)}^{-}(c, b)(f, d)<\sigma$ then since $\omega_{(c, d)}^{-}(f, d)<\left(\omega^{\prime}\right)_{(c, d),(f, b)}^{-}(c, b)(f, d)$ we get that both predecessors of $\omega$ connected to $(c, d)$ and $f$ are in the graph, in contradiction with the assumption. 
- If $v_{(a, b)}^{-}(d, b)<\sigma$ recall that $\omega^{\prime}=\omega_{(a, b)}^{-}(a, f)<\sigma$ so that

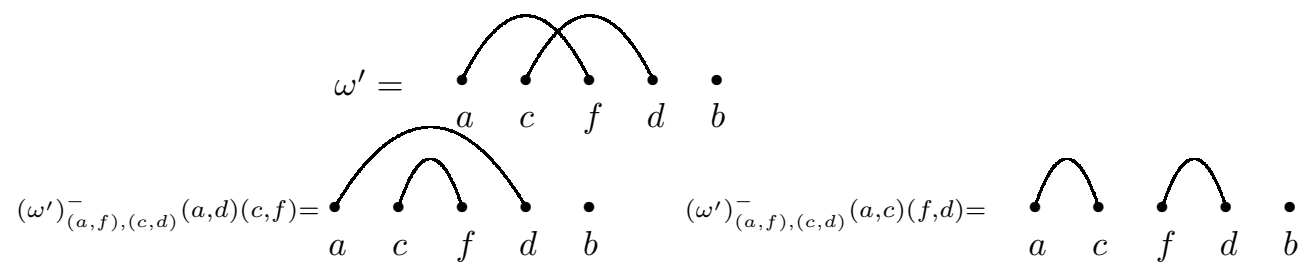

Exactly as in the first case,

if $\left(\omega^{\prime}\right)_{(a, f),(c, d)}^{-}(a, d)(c, f)$ then $\omega<v$ satisfying $v_{(a, b)}^{-}(a, d), v_{(a, b)}^{-}(d, b)<\sigma$ so that $\omega \notin$ $\operatorname{Sing}(\sigma)$;

if $\left(\omega^{\prime}\right)_{(a, f),(c, d)}^{-}(a, c)(f, d)<\sigma$ then both predecessors of $\omega$ connected to $(c, d)$ and $f$ are in the graph, in contradiction with the assumption.

So there cannot be $(c, d) \in \omega$ such that $a<c<f<d<b$.

Now, let us show that for $(c, d) \in S$ one has $\omega_{(c, d)}^{-}(c, f), \omega_{(c, d)}^{-}(f, d)<\sigma$. Indeed, since $\omega_{(a, b)}^{-}(a, f)<$ $\sigma$ one of its predecessors $\omega_{(a, b),(c, d)}^{-}(a, f)(c, b), \omega_{(a, b),(c, d)}^{-}(a, f)(b, d)$ is in $G_{\sigma}$. Exactly in the same way, since $\omega_{(a, b)}^{-}(f, b)<\sigma$ one of its predecessors $\omega_{(a, b),(c, d)}^{-}(f, b)(c, a), \omega_{(a, b),(c, d)}^{-}(f, b)(a, d)$ is in $G_{\sigma}$. Note that

(i) $\omega_{(c, d)}^{-}(c, f)<\omega_{(a, b),(c, d)}^{-}(a, f)(c, b)$ so that if $\omega_{(a, b),(c, d)}^{-}(a, f)(c, b) \leq \sigma$ then $\omega_{(c, d)}^{-}(c, f)<\sigma$;

(ii) $\omega_{(c, d)}^{-}(f, d)<\omega_{(a, b),(c, d)}^{-}(a, f)(b, d)$ so that if $\omega_{(a, b),(c, d)}^{-}(a, f)(b, d) \leq \sigma$ then $\omega_{(c, d)}^{-}(f, d)<\sigma$;

(iii) $\omega_{(c, d)}^{-}(c, f)<\omega_{(a, b),(c, d)}^{-}(c, a)(f, b)$ so that if $\omega_{(a, b),(c, d)}^{-}(c, d)(f, b) \leq \sigma$ then $\omega_{(c, d)}^{-}(c, f)<\sigma$;

(iv) $\omega_{(c, d)}^{-}(f, d)<\omega_{(a, b),(c, d)}^{-}(a, d)(f, b)$ so that if $\omega_{(a, b),(c, d)}^{-}(a, d)(f, b) \leq \sigma$ then $\omega_{(c, d)}^{-}(f, d)<\sigma$;

Thus if either $\omega_{(a, b),(c, d)}^{-}(a, f)(c, b), \omega_{(a, b),(c, d)}^{-}(a, d)(f, b) \in G_{\sigma}$ or $\omega_{(a, b),(c, d)}^{-}(a, f)(b, d), \omega_{(a, b),(c, d)}^{-}(c, a)(f, b) \in G_{\sigma}$ then both $\omega_{(c, d)}^{-}(c, f), \omega_{(c, d)}^{-}(f, d)<\sigma$. Let us show that it cannot occur that either

$$
\omega_{(a, b),(c, d)}^{-}(a, f)(c, b), \omega_{(a, b),(c, d)}^{-}(c, a)(f, b) \leq \sigma
$$

or

$$
\omega_{(a, b),(c, d)}^{-}(a, f)(b, d), \omega_{(a, b),(c, d)}^{-}(a, d)(f, b) \leq \sigma .
$$

Indeed, if

$$
\omega_{(a, b),(c, d)}^{-}(a, f)(c, b), \omega_{(a, b),(c, d)}^{-}(c, a)(f, b) \leq \sigma
$$

then $\omega_{(c, d)}^{-}(c, f) \in G_{\sigma}$ and both

$$
\omega_{(a, b),(c, d)}^{-}(a, f)(c, b), \omega_{(a, b),(c, d)}^{-}(c, a)(f, b)
$$


are its predecessors, obtained from "uncrossing" $(c, f),(a, b)$ so that $\mathcal{F}_{\omega_{(a, b),(c, d)}^{-}(c, f)(a, b)}$ is singular in $\mathcal{F}_{\sigma}$ but $\omega_{(c, d)}^{-}(c, f)$ is a predecessor of $\omega$, which contradicts to $\omega \in \operatorname{Sing}(\sigma)$. Exactly in the same way if $\omega_{(a, b),(c, d)}^{-}(a, f)(b, d), \omega_{(a, b),(c, d)}^{-}(a, d)(f, b) \leq \sigma$ then $\omega_{(c, d)}^{-}(f, d) \in G_{\sigma}$ and both $\omega_{(a, b),(c, d)}^{-}(a, f)(b, d), \omega_{(a, b),(c, d)}^{-}(a, d)(f, b)$ are its predecessors obtained from "uncrossing" $(a, b),(f, d)$ which again contradicts to $\omega \in \operatorname{Sing}(\sigma)$.

Note that in our proof that $(c, d) \in S$ cannot cross $(a, b)$ we used only fact that both

$$
\omega_{(a, b)}^{-}(a, f), \omega_{(a, b)}^{-}(f, b)<\sigma
$$

but not its minimality, so that this is true for any $(i, j),\left(i^{\prime}, j^{\prime}\right) \in S$ and all of them are concentric. Consider $f \neq p \in \omega^{0} \cap[a, b]$, let us show that $\omega_{(a, b)}^{-}(a, p), \omega_{(a, b)}^{-}(p, b)<\sigma$ and $\omega_{(a, b)}^{-}(p, f) \nless \sigma$. We can assume that $p<f$ then since $\omega_{(a, b)}^{-}(p, b)<\omega_{(a, b)}^{-}(f, b)$ we get that $\omega_{(a, b)}^{-}(p, b)<\sigma$. Now since $a<p<f$ one has either $\omega_{(a, b)}^{-}(a, p)<\sigma$ or $\omega_{(a, b)}^{-}(p, f)<\sigma$. If $\omega_{(a, b)}^{-}(p, f)<\sigma$ then for $\omega^{\prime}=\omega_{(a, b)}^{-}(p, b)$ both predecessors $\left(\omega^{\prime}\right)_{(p, b)}-(p, f),\left(\omega^{\prime}\right)_{(p, b)}-(f, b)<\sigma$ so that $\mathcal{F}_{\omega^{\prime}}$ is singular in $\mathcal{F}_{\sigma}$ in contradiction with $\omega \in \operatorname{Sing}(\sigma)$.

To finish with (ii), let us show that if there exists $(c, d) \in S$ and $p \in \omega^{0}$ such that $p \in[c, d] \backslash[a, b]$, then $\omega \notin \operatorname{Sing}(\sigma)$. Without loss of generality, we can assume that $p \in(c, a)$. Then by Proposition 17 either $\omega_{(c, d)}^{-}(c, p)$ or $\omega_{(c, d)}^{-}(p, d)$ is in $G_{\sigma}$.

(a) If $\omega_{(c, d)}^{-}(c, p) \in G_{v}$, then noting that both $\omega_{(a, b)}^{-}(a, f)$ and $\omega_{(a, b)}^{-}(f, b)$ are in $G_{\sigma}$, we get that $\sigma>\omega_{(c, d)(a, b)}^{-}(c, p)(a, f), \omega_{(c, d)(a, b)}^{-}(c, p)(f, b)$ so that $\omega_{(c, d)}^{-}(c, p)$ is singular in $G_{v}$ and $\omega>\omega_{(c, d)}^{-}(c, p)$ is not in $\operatorname{Sing}(\sigma)$.

(b) If $\omega_{(c, d)}^{-}(p, d) \in G_{v}$, then noting that on one hand $\omega_{(c, d)}^{-}(f, d)=\left(\omega_{(c, d)}^{-}(p, d)\right)_{(p, d)}^{-}(f, d) \in G_{\sigma}$. On the other hand, one of the predecessors of $\omega_{(c, d)}^{-}(c, f)$ connected to $p$ must be in $G_{\sigma}$. Note that $\left(\omega_{(c, d)}^{-}(c, f)\right)_{(c, f)}^{-}(c, p)=\omega_{(c, d)}^{-}(c, p)$ so we return to case $(\mathrm{a})$, or $\left(\omega_{(c, d)}^{-}(c, f)\right)_{(c, f)}^{-}(p, f)=$ $\left(\omega_{(c, d)}^{-}(p, d)\right)_{(p, d)}^{-}(p, f)$ so that both predecessors of $\omega_{(c, d)}^{-}(p, d)$ connected to $f$ are in $G_{\sigma}$, which implies $\omega \notin \operatorname{Sing}(\sigma)$, again.

(iii) Note first of all that if $(i, j) \notin S$, then $(i, j)$ cannot be a bridge over $p \in \omega^{0} \cap[a, b]$, by (ii). Now, again, we have to consider the following cases:

(a) there is $p \in \omega^{0} \backslash[a, b]$ and there is $(i, j) \in \omega$ such that $i<p<j$. In this case, we show that there exists $\omega^{\prime}>\omega$ such that $\omega^{\prime}$ is singular in $G_{\sigma}$.

(b) there exist $(i, j),\left(i^{\prime}, j^{\prime}\right) \in \omega \backslash S$ such that $i<i^{\prime}<j<j^{\prime}$. Again, in this case, we show that there exists $\omega^{\prime}>\omega$ such that $\omega^{\prime}$ is singular in $G_{\sigma}$.

(c) there is $(i, j) \notin S$ and $(c, d),(a, b) \in S$ with $c<a<b<d$ such that either $(i, j)$ crosses $(c, d)$ but not $(a, b)$ or $(i, j)$ crosses $(a, b)$ but not $(c, d)$. In this case, we show that there exists $\omega^{\prime}>\omega$ such that $\omega^{\prime}$ is singular in $G_{\sigma}$. 
Exactly as in (ii) all the cases are analyzed in the same way, so we show only case (a). Assume that there is a fixed point $p$ under $(i, j) \in \omega \backslash S$. Since any fixed point at $[a, b]$ has the same role as $f$, and there are no fixed points under $(c, d) \in S$ such that they are not at $[a, b]$, by (ii) we get that $p$ is not under any arc of $S$. Thus, without loss of generality, we can assume that $i<p<c$ for any $(c, d) \in S$.

As in the proof of (ii), we see that at least one of

$$
\omega_{(i, j)}^{-}(i, p), \omega_{(i, j)}^{-}(p, j)
$$

is smaller than $\sigma$. We have either $i<p<j<a$ or $i<p<a<j<f$, where $f$ is the most left fixed point at $[a, b]$. So let us consider these two cases:

(a1) Case $i<p<j<a$. We have

$$
\omega_{(a, b)}^{-}(a, f), \omega_{(a, b)}^{-}(f, b) \in G_{\sigma}
$$

and either $\omega_{(i, j)}^{-}(i, p)$ or $\omega_{(i, j)}^{-}(p, j)$ in $G_{\sigma}$. Note that

$$
\left(R_{\sigma}\right)_{i, p} \geq\left(R_{\omega}\right)_{i, p}+1 \text { or }\left(R_{\sigma}\right)_{p, j} \geq\left(R_{\omega}\right)_{p, j}+1
$$

and

$$
\left(R_{\sigma}\right)_{a, f} \geq\left(R_{\omega}\right)_{a, f}+1 \text { and }\left(R_{\sigma}\right)_{f, b} \geq\left(R_{\omega}\right)_{f, b}+1
$$

and $[i, p] \cap[a, f]=\emptyset,[p, j] \cap[a, f]=\emptyset$, respectively. Thus

$$
v=\left\{\begin{array}{r}
\omega_{0}(i, p)(a, f), \omega_{0}(i, p)(f, b) \in G_{\sigma} \\
\text { if } \omega^{-}(i, j)(i, p) \in G_{\sigma} \\
\omega_{0}(p, j)(a, f), \omega_{0}(p, j)(f, b) \in G_{\sigma} \\
\text { if } \omega_{(i, j)}^{-}(p, j) \in G_{\sigma}
\end{array}\right.
$$

So $\omega_{(i, j)}^{-}(i, p)$ or $\omega_{(i, j)}^{-}(p, j)$ is singular in $G_{\sigma}$, respectively. Hence, $\omega \notin \operatorname{Sing}(\sigma)$.

(a2) Case $i<p<a<j<f$. If $\omega_{(i, j)}^{-}(i, p) \in G_{\sigma}$, then exactly as in Case (a1), we get that $\omega_{(i, j)}^{-}(i, p)$ is singular in $G_{\sigma}$, so that $\omega \notin \operatorname{Sing}(\sigma)$. Now, let $\omega_{(i, j)}^{-}(p, j) \in G_{\sigma}$ :

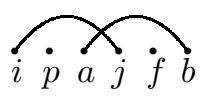

Note that $R_{\omega}=R_{0}+R_{(i, j)(a, b)}$ and the corresponding part of $R_{(i, j)(a, b)}$ at $[i, b]$ (not taking into account other entries) is 


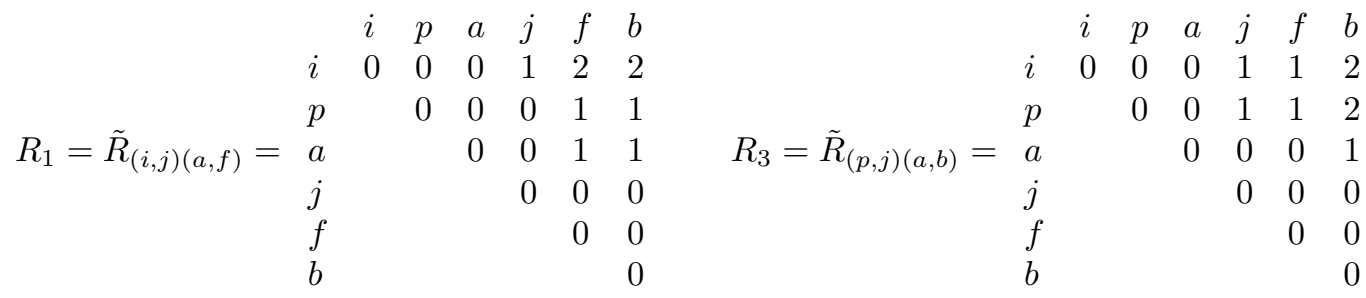

$$
\begin{aligned}
& \begin{array}{ccccccc} 
& i & p & a & j & f & b \\
i & 0 & 0 & 0 & 1 & 1 & 2 \\
p & & 0 & 0 & 0 & 0 & 1 \\
R_{2}=\tilde{R}_{(i, j)(f, b)}= & & & 0 & 0 & 0 & 1 \\
j & & & & 0 & 0 & 1 \\
f & & & & & 0 & 1 \\
b & & & & & & 0
\end{array}
\end{aligned}
$$

Now, let $(M)_{s, t}=\max \left\{\left(R_{1}\right)_{s, t},\left(R_{3}\right)_{s, t}\right\}$, we get

$$
M:=\tilde{R}_{(i, j)(f, b)}=\begin{array}{ccccccc} 
& i & p & a & j & f & b \\
i & 0 & 0 & 0 & 1 & 2 & 2 \\
p & & 0 & 0 & 1 & 1 & 2 \\
a & & & 0 & 0 & 1 & 1 \\
j & & & & 0 & 0 & 0 \\
f & & & & & 0 & 0 \\
b & & & & & & 0
\end{array}
$$

is not a rank matrix but since $M_{p, j}=1$ and $M_{a, f}=1$, we get that $\omega_{0}(p, j)(a, f) \in G_{\sigma}$.

Now, let $(N)_{s, t}=\max \left\{\left(R_{2}\right)_{s, t},\left(R_{3}\right)_{s, t}\right\}$, we get

$$
N:=\tilde{R}_{(i, j)(f, b)}=\begin{array}{ccccccc} 
& i & p & a & j & f & b \\
i & 0 & 0 & 0 & 1 & 1 & 2 \\
p & & 0 & 0 & 1 & 1 & 2 \\
a & & & 0 & 0 & 0 & 1 \\
j & & & & 0 & 0 & 1 \\
f & & & & & 0 & 1 \\
b & & & & & & 0
\end{array}
$$

where $N$ is a rank matrix of $\omega_{0}(f, b)(p, j)$ so it is in $G_{\sigma}$. So, $\omega_{(i, j)}^{-}(p, j)$ is in $G_{\sigma}$ and $\omega \notin$ $\operatorname{Sing}(\sigma)$.

This completes the proof.

As a corollary we get the following proposition

Proposition 21. Let $\sigma \in I_{n, k}^{\max }$ be such that $1, n \in \sigma^{0}$ and $\left|\tau^{*}(\sigma)\right| \geq 2$. Let $\omega \in \operatorname{Sing}(\sigma)$. If there exists $f \in \omega^{0}$ and $(i, j) \in \omega$ such that $\omega_{(i, j)}^{-}(i, f), \omega_{(i, j)}^{-}(f, j)<\sigma$ then $\omega$ is obtained by our algorithm. 
Proof. We prove it by induction on $n$ starting with $n=6$ and $\sigma=(2,3)(4,5)$, which is known to be true.

Let $(a, b) \in \omega$ be the minimal bridge over $f$. By Lemma $20 \pi_{a+1, b-1}(\omega)$ either equal to $\pi_{a+1, b-1}(\sigma)$ or is obtained from $\pi_{a+1, b-1}(\sigma)$ by deleting some external arcs. Let us show, that if some external arc of $\pi_{a+1, b-1}(\sigma)$ is deleted then $\omega \notin \operatorname{Sing}(\sigma)$. First of all note that $f \in\left(\pi_{a+1, b-1} \sigma\right)^{0}$. Indeed, if it is not a fixed point then either there exists $(i, f) \in \sigma$ where $i \geq a+1$ or $(f, j) \in \sigma$ where $j \leq b-1$. We can assume $(i, f) \in \sigma$, but then $i$ is a fixed point of $\omega$ and then $\omega_{(a, b)}^{-}(i, b)$ satisfies $\omega_{(a, b)}^{-}(i, f), \omega_{(a, b)}^{-}(f, b)<\sigma$ in contradiction to $\omega \in \operatorname{Sing}(\sigma)$. Thus, $f \in\left(\pi_{a+1, b-1}(\sigma)\right)^{0}$. Now, if $\pi_{a+1, b-1}(\omega) \neq \pi_{a+1, b-1}(\sigma)$ then there exists $(i, j) \in \pi_{a+1, b-1}(\sigma)$ such that $(i, j) \notin \pi_{a+1, b-1}(\omega)$, and by maximality of $\pi_{a+1, b-1}(\sigma)$ either $j<f$ or $i>f$. We can assume that $j<f$ and then we get again $\omega_{(a, b)}^{-}(i, b)$ satisfies $\omega_{(a, b)}^{-}(i, f), \omega_{(a, b)}^{-}(f, b)<\sigma$ in contradiction to $\omega \in \operatorname{Sing}(\sigma)$. Thus, $\pi_{a+1, b-1}(\omega)=\pi_{a+1, b-1}(\sigma)$. Now consider $\pi_{a, b}(\sigma)$. Since $f$ is a fixed point of $\pi_{a+1, b-1}(\sigma)$ one has $(a, b) \notin \pi_{a, b}(\sigma)$. Moreover, since $\pi_{a+1, f-1}(\sigma)=\pi_{a+1, f-1}(\omega)$ and $R_{a, f}(\omega)=R_{a+1, f-1}(\omega) \leq$ $R_{a, f}(\sigma)-1$ one has by maximality of $\sigma$ that $(a, i) \in \sigma$ where $i=\min \left(\pi_{a+1, b-1}(\sigma)\right)^{0}$ and obviously $i \leq f$. Exactly in the same way $(j, b) \in \sigma$ where $j=\max \left(\pi_{a+1, b-1}(\sigma)\right)^{0}$ and $j \geq\{f, i+1\}$. Then one has $\pi_{a, b}(\omega)=\left(\pi_{a, b}(\sigma)\right)_{(a, i),(j, b)}^{-}(a, b)$.

Moreover if $\pi_{a+1, b-1}(\sigma) \neq \emptyset$ then there exists $(i, i+1) \in \sigma, \omega$ and then by Lemma 19 and by induction $\omega$ is obtained from $\sigma$ by our algorithm.

Thus we are left with the case $\pi_{a, b}(\sigma)=(a, a+1)(b-1, b)$ and $\pi_{a, b}(\omega)=(a, b)$. Further,

- either $b>a+3$ then if either $(a-1, a+2) \in \sigma$ or $(b-2, b+1) \in \sigma$ we can assume $(a-1, a+2) \in \sigma$ then $\pi_{a-1, b}(\omega)=(a-1, a+1)(a, b)$ and let $v=\omega_{(a-1, a+1),(a, b)}^{-}(a-1, b)(a, a+1)>\omega$ and $v_{(a-1, b)}^{-}(a-1, f), v_{(a-1, b)}(f, b)<\sigma$, so that $\omega \notin \operatorname{Sing}(\sigma)$. Thus, if $b>a+3$ we get that $a-1, b+1 \in\left(\pi_{a-1, b+1}(\sigma)\right)^{0}$. If $(a-1, b+1) \in \omega$ then $R_{a-1, b+1}(\omega)=2=R_{a-1, b+1}(\sigma)$ and then $\omega$ is obtained by our algorithm, either by induction since at least one of $1, n$ is a fixed point of $\omega$ or $a-1=1, b+1=n$ and then straightforwardly.

- or $b=a+3$. If $\sigma=(2, n-1)(3, n-2) \ldots(a-1, a+4)(a, a+1)(a+2, a+3)$ then $\omega=$ $(1, n) \ldots(a-1, a+4)(a, a+3)$ by straightforward computation. Otherwise, there exists $(i, i+1) \in \sigma$ where $i<a$ or $i>b$. We can assume $i<a$. Again a straightforward computation shows either $(i, i+1) \in \omega$ or $1 \in \omega^{0}$. In both cases we get the result by induction.

In order to finish the proof we have to show

Proposition 22. Let $\sigma \in I_{n, k}^{\max }$ be such that $1, n \in \sigma^{0}$ and let $\omega \in \operatorname{Sing}(\sigma)$ be such that there is no $f \in \omega^{0}$ and $(a, b) \in \omega$ such that

$$
a<f<b \text { and } \omega_{(a, b)}^{-}(a, f), \omega_{(a, b)}^{-}(f, b)<\sigma
$$

then $b(\omega)=0$. In particular, $1, n \in \omega^{0}$ and $\pi_{2, n-1}(\omega) \in \operatorname{Sing}\left(\pi_{2, n-1}(\sigma)\right)$ so that $\omega$ is obtained by our algorithm. 
Proof. Since $\omega \in \operatorname{Sing}(\sigma)$ and there is no $f \in \omega^{0}$ and $(a, b) \in \omega$ such that $a<f<b$ and $\omega_{(a, b)}^{-}(a, f)$, $\omega_{(a, b)}^{-}(f, b)<\sigma$, there exist $(a, c),(b, d) \in \omega$ where $a<b<c<d$ such that $\omega_{(a, c),(b, d)}^{-}(a, b)(c, d)$, $\omega_{(a, c),(b, d)}^{-}(a, d)(b, c) \leq \sigma$ and for both of them $\mathcal{F}_{\omega_{(a, c),(b, d)}^{-}(a, b)(c, d)}, \mathcal{F}_{\omega_{(a, c),(b, d)}^{-}(a, d)(b, c)}$ are smooth in $\mathcal{F}_{\sigma}$. Assume there exists $f \in \omega^{0}$ and $(i, j) \neq(a, c),(b, d)$ such that $i<f<j$ then the same is true for $\omega_{(a, c),(b, d)}^{-}(a, b)(c, d), \omega_{(a, c),(b, d)}^{-}(a, d)(b, c)$. Thus exactly one out of

$$
\left.\left.\omega_{(a, c),(b, d),(i, j)}^{-}(a, b)(c, d)\right)(i, f), \omega_{(a, c),(b, d),(i, j)}^{-}(a, b)(c, d)\right)(f, j)
$$

is in $G_{\sigma}$ and exactly one out of

$$
\left.\left.\omega_{(a, c),(b, d),(i, j)}^{-}(a, d)(b, c)\right)(i, f), \omega_{(a, c),(b, d),(i, j)}^{-}(a, d)(b, c)\right)(f, j)
$$

is in $G_{\sigma}$. If it is the same one, for example

$$
\left.\left.\omega_{(a, c),(b, d),(i, j)}^{-}(a, b)(c, d)\right)(i, f), \omega_{(a, c),(b, d),(i, j)}^{-}(a, d)(b, c)\right)(i, f)
$$

then $\omega_{(i, j)}^{-}(i, f)$ has 2 predecessors in $G_{\sigma}$ connected to "uncrossing" $(a, c),(b, d)$ in contradiction to $\omega \in \operatorname{Sing}(\sigma)$ If they are different, for example

$$
\omega_{(a, c),(b, d),(i, j)}^{-}(a, b)(c, d)(i, f), \omega_{(a, c),(b, d),(i, j)}^{-}(a, d)(b, c)(f, j)
$$

is in $G_{\sigma}$ then

$$
\begin{aligned}
& \left.\omega_{(i, j)}^{-}(i, f)<\omega_{(a, c),(b, d),(i, j)}^{-}(a, b)(c, d)\right)(i, f), \\
& \left.\omega_{(i, j)}^{-}(f, j)<\omega_{(a, c),(b, d),(i, j)}^{-}(a, d)(b, c)\right)(f, j)
\end{aligned}
$$

so that both predecessors connected to $(i, j)$ and $f$ are in $G_{\sigma}$ in contradiction with the conditions. Now assume $a<f<b$, put $\omega^{\prime}=\omega_{(a, c),(b, d)}^{-}$then one of $\omega^{\prime}(f, b)(c, d), \omega^{\prime}(a, f)(c, d)<\sigma$ and one of $\omega^{\prime}(f, d)(b, c), \omega^{\prime}(a, f)(b, c)$ is in $G_{\sigma}$.

- If $\omega^{\prime}(a, f)(c, d), \omega^{\prime}(a, f)(b, c)<\sigma$ then they are both predecessors of $\omega^{\prime}(a, f)(b, d)>\omega$ in contradiction to $\omega \in \operatorname{Sing}(\sigma)$;

- If $\omega^{\prime}(a, f)(c, d), \omega^{\prime}(f, d)(b, c)<\sigma$ then by maximality of $\sigma$ exactly as in the proof of Lemma 20 $\omega^{\prime}(f, c)(b, d)$ has 2 predecessors in $G_{\sigma}$ connected to "uncrossing" $(f, c)(b, d)$ in contradiction to $\omega \in \operatorname{Sing}(\sigma)$;

- If $\omega^{\prime}(f, b)(c, d), \omega^{\prime}(a, f)(b, c)<\sigma$ then again, exactly as in the proof of of Lemma 20 by maximality of $\sigma$ one has $\omega^{\prime}(f, c)(b, d)$ has two predecessors connected to "uncrossing" $(f, c),(b, d)$ in $G_{\sigma}$ in contradiction to $\omega \in \operatorname{Sing}(\sigma)$;

- If $\omega^{\prime}(f, b)(c, d), \omega^{\prime}(f, d)(b, c)<\sigma$ then $\omega^{\prime}(f, c)(b, d)$ has two predecessors connected to "uncrossing" $(f, c),(b, d)$ in $G_{\sigma}$ in contradiction to $\omega \in \operatorname{Sing}(\sigma)$;

Thus there cannot be fixed points at $[a, b],[c, d]$. The last case is there exists $f \in \omega^{0} \cap[b, c]$. In this case by symmetry it is enough to to consider case $\omega^{\prime}(b, f)(a, d)<\sigma$. Then for $\omega^{\prime}(a, f)(b, d)$ one has $\omega^{\prime}(a, b)(f, d)<\omega^{\prime}(a, b)(c, d)<\sigma$ and $\omega^{\prime}(a, d)(b, f)<\sigma$ and $\omega^{\prime}(a, b)(f, d)>\omega$ in contradiction to $\omega \in \operatorname{Sing}(\sigma)$. 
Thus there are no fixed points under arcs of $\omega$. If $1 \notin \omega^{0}$ then let $i=\min \omega^{0}$. One has $i$ is odd and $\left(R_{\omega}\right)_{1, i-1}=0.5(i-1)$. Since 1 is fixed point of $\sigma$ one has $\left(R_{\sigma}\right)_{1, i-1}=\left(R_{\sigma}\right)_{2, i-1}<0.5(i-1)$ which contradicts $\omega<\sigma$. Thus both $1, n$ are fixed points of $\omega$.

As an immediate corollary we get

Corollary 23. For all $\sigma \in I_{n, k}^{\max }$ with $\rho(\sigma) \geq 4$, and $v<\sigma$ such that $\operatorname{codim}_{\mathcal{F}_{\sigma}} \mathcal{Z}_{v} \leq 3$ one has $\mathcal{F}_{v}$ is smooth in $\mathcal{F}_{\sigma}$ and $v \in \operatorname{Sing}(\sigma)$ satisfies

$$
\operatorname{dim} \mathcal{T}_{\mathcal{F}_{\sigma}}\left(\mathcal{F}_{v}\right)=\operatorname{dim} \mathcal{F}_{\sigma}+\operatorname{codim}_{\mathcal{F}_{\sigma}}\left(\mathcal{F}_{v}\right)
$$

Remark: There exists non-maximal $\sigma \in I_{n, k}$ and $v \in \operatorname{Sing}(\sigma)$ such that $\operatorname{codim}_{\mathcal{F}_{\sigma}} \mathcal{F}_{v}=3$, but a straightforward computation shows that for any $\sigma \in I_{n, k}$ and $v \in G_{\sigma}$ such that $\operatorname{codim}_{\mathcal{F}_{\sigma}} \mathcal{F}_{v} \leq 2$, $\mathcal{F}_{v}$ is smooth in $\mathcal{F}_{\sigma}$. This is known to be true for $v$ of $\operatorname{codim}_{\mathcal{F}_{\sigma}} \mathcal{F}_{v}=1$, see [8]. For $\operatorname{codim}_{\mathcal{F}_{\sigma}} \mathcal{F}_{v}=2$, one can compute this combinatorially.

\section{Remarks on singular locus of $\mathcal{F}_{\sigma}$ for $\sigma \in I_{n, k}^{\max }$}

Note that the number of components depends heavily on the structure of $\sigma$ even for $\sigma \in I_{n, k}^{\max }$ where our algorithm provides all $v \in \operatorname{Sing}(\sigma)$. We can only note that for $\sigma \in I_{n, k}^{\max }$ with $\rho(\sigma) \geq 4$ the number of components is greater or equal to $\left(\begin{array}{c}\rho(\sigma)-2 \\ 2\end{array}\right)$.

Indeed, we can assume that $\sigma$ is a link pattern with $1, n \in \sigma^{0}$ and $\left|\tau^{*}(\sigma)\right| \geq 2$. Then, by our algorithm in the beginning of 4.3 each pair $i, j \in \tau^{*}(\sigma)$ gives rise to the pair of admissible arcs at least at one interval, and for $\{i, j\} \neq\left\{i^{\prime}, j^{\prime}\right\}$ we get different pairs of admissible arcs.

In particular we get

Proposition 24. For $\sigma \in I_{n, k}^{\max }$ with $\rho(\sigma)>4$ the number of elements in Sing $(\sigma)$ is greater or equal to 3.

In order to describe the picture in the case $\sigma \in I_{n, k}^{\max }$ with $\rho(\sigma)=4$ recall notion of $\sigma_{+a}$, complete link pattern and concatenation from Subsection 3.2 Let $\operatorname{con}(k)=(1,2 k)(2,2 k-1) \cdots(k, k+1)$ denote a link pattern with $2 k$ points and $k$ concentric arcs. Recall that each maximal link pattern can be written as a concatenation of maximal complete link patterns and fixed points. For instance, the link pattern $(2,3)(5,8)(6,7)(11,16)(12,13)(14,15) \in I_{18,6}$ is maximal and can be written as $\left(\operatorname{con}(1)_{+1}\right)\left(\operatorname{con}(2)_{+4}\right)\left(\sigma_{+10}^{\prime}\right)$, where $\operatorname{con}(1), \operatorname{con}(2)$ and $\sigma^{\prime}=(1,6)(2,3)(4,5)$ are maximal complete link patterns.

Let $\sigma=\left(\operatorname{con}\left(k_{1}\right)_{+a_{1}}\right)\left(\operatorname{con}\left(k_{2}\right)_{+a_{2}}\right) \cdots\left(\operatorname{con}\left(k_{m}\right)_{+a_{m}}\right) \in I_{n, s}$ be a maximal link pattern where $a_{1} \geq 1$, $a_{i} \geq a_{i-1}+2 k_{i-1}+1, s=\sum_{j=1}^{m} k_{j}$ and $n \geq a_{m}+2 k_{m}+1$, that is the union of sets of concentric arcs where each set is separated from the next one by nonempty set of fixed points and $1, n$ are fixed points. Then the number of components of $\sigma$ is exactly $\left(\begin{array}{c}m \\ 2\end{array}\right)$. In particular, we get

Proposition 25. Let $\sigma \in I_{n, k}^{\max }$ be such that $\rho(\sigma)=4$. Then Sing $(\sigma)$ contains the unique element.

Proof. Note that $\sigma$ is a completion of $\sigma^{\prime}$ where there are only two possibilities for $\sigma^{\prime}$ : 


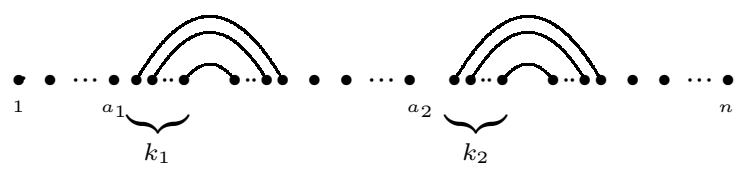

- $\sigma^{\prime}=\left(\operatorname{con}\left(k_{1}\right)_{+a_{1}}\right)\left(\operatorname{con}\left(k_{2}\right)_{+a_{2}}\right) \in I_{n, k_{1}+k_{2}}$ with $a_{1} \geq 1, a_{2} \geq 2 k_{1}+a_{1}+1$ and $n \geq a_{2}+2 k_{2}+1$ : In this case the unique admissible pair is $\left(a_{1}, 2 k_{1}+a_{1}-1\right),\left(a_{2}, 2 k_{2}+a_{2}-1\right)$ and it is admissible at the unique interval $\left[a_{1}, 2 k_{2}+a_{2}+1\right]$.

- $\sigma^{\prime}=\left(i+1,2\left(k_{1}+k_{2}+k_{3}\right)+i\right)\left(i+2,2\left(k_{1}+k_{2}+k_{3}+i-1\right)\right) \cdots\left(i+k_{3}, i+k_{3}+2\left(k_{1}+k_{2}\right)+\right.$ 1) $\left(\operatorname{con}\left(k_{1}\right)_{+\left(i+k_{3}\right)}\right)\left(\operatorname{con}\left(k_{2}\right)_{+\left(i+k_{3}+2 k_{1}\right)}\right) \in I_{n, k_{1}+k_{2}+k_{3}}$ such that $i \geq 1, k_{1}, k_{2} \geq 1, k_{3} \geq 0$ and $n>i+2\left(k_{1}+k_{2}+k_{3}\right)$.

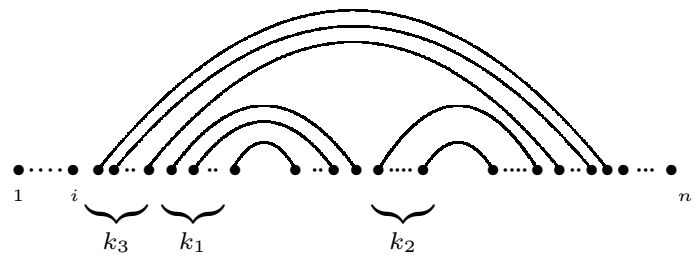

In this case the only admissible pair is $\left(i+k_{3}+1, i+k_{3}+2 k_{1}\right),\left(i+k_{3}+2 k_{1}+1, i+k_{3}+2 k_{1}+2 k_{2}\right)$ and it is admissible at the unique the interval $\left[i, i+2\left(k_{1}+k_{2}+k_{3}\right)+1\right]$.

If $\sigma \in I_{n, k}$ is non maximal, the picture is more complex. We would like to finish the paper with providing an example of a singular $Z_{x}$-orbit inside a smooth component.

Example 26. Consider an example in $s \ell_{8}(\mathbb{C})$ : Let $\mathcal{Z}_{\omega}$ be a $Z$-orbit with $\omega=(1,4)(2,7)(3,6)(5,8)$, and it is the component of singularity of $\mathcal{F}_{T}$ where

$$
T=\begin{array}{|l|l|}
\hline 1 & 2 \\
\hline 3 & 4 \\
\hline 5 & 6 \\
\hline 7 & 8 \\
\hline
\end{array}
$$

with the corresponding link pattern $\sigma_{T}=(1,2)(3,4)(5,6)(7,8)$. The corresponding admissible pair is $(3,4)(5,6)$ and interval $[2,7]$. Note that $\mathcal{Z}_{\omega}$ is also a $Z$-orbit in $\mathcal{F}_{S}$, where

$$
S=\begin{array}{|l|l|}
\hline 1 & 4 \\
\hline 2 & 6 \\
\hline 3 & 7 \\
\hline 5 & 8 \\
\hline
\end{array}
$$

with the corresponding link pattern $\sigma_{S}=(1,8)(2,7)(3,4)(5,6)$. Since $\mathcal{F}_{S}$ is smooth, so $\mathcal{Z}_{\omega}$ is a smooth $\mathcal{Z}$-orbit in it (which is not surprising since the singularity of $Z$-orbit $\mathcal{Z}^{\prime}$ in the closure of 
another $Z$-orbit $\mathcal{Z}$ depends on both $\mathcal{Z}$ and $\left.\mathcal{Z}^{\prime}\right)$. However, $\mathcal{Z}_{\omega}$ is the component of singularity in $\overline{\mathcal{Z}}_{v}$ with $v=(1,7)(2,8)(3,4)(5,6)$. One see at once that (1) $\mathcal{Z}_{v} \subset \mathcal{F}_{S}$ (of $\operatorname{codim}_{\mathcal{F}_{S}} \mathcal{F}_{v}=1$ ), (2) $\mathcal{Z}_{\omega} \subset \overline{\mathcal{Z}}_{v},(3)$ codim $\overline{\mathcal{Z}}_{v} \mathcal{Z}_{\omega}=3$. As for $\operatorname{dim} \mathcal{T}_{F_{\omega}}\left(\mathcal{F}_{v}\right)$ one can see that there are 4 predecessors of $\omega$ in $G_{v}$, see Figure 5 .

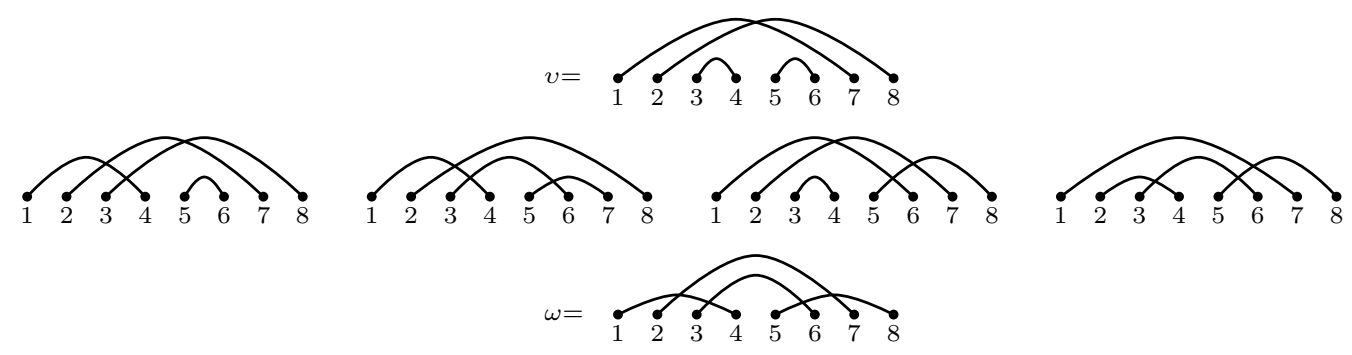

Figure 5: The link patterns $\omega$ and $v$, and the predecessors of $\omega$ in $G_{L_{S}}$.

One can also note at once that these are the predecessors of $\omega$ in $G_{\sigma_{S}}$. Thus $\operatorname{dim} \mathcal{T}_{F_{\omega}}\left(\mathcal{F}_{v}\right)=$ $\operatorname{dim} \mathcal{T}_{F_{\omega}}\left(\mathcal{F}_{S}\right)=\operatorname{dim} \mathcal{F}_{S}=\operatorname{dim} \mathcal{Z}_{v}+1$

This example provides us with three facts:

- A singular component in $\mathcal{Z}$ can be of codimension 3.

- $\operatorname{dim} \mathcal{T}_{\overline{\mathcal{Z}}}\left(\mathcal{Z}^{\prime}\right)-\operatorname{dim} \mathcal{Z}$ can be smaller than $\operatorname{codim}_{\overline{\mathcal{Z}}} \mathcal{Z}^{\prime}$ (indeed here it is $\operatorname{dim} \mathcal{Z}+1$ ).

- In a smooth component there are singular $\mathcal{Z}$-orbit closures.

\section{Notation}

$\S 1 \quad \mathbb{K}, V, \mathcal{F}$

$\S 1.1 x, \mathcal{F}_{x}$

$\S 1.2 \quad \lambda, \lambda \vdash n, Y(x)=Y_{\lambda}, \lambda^{*}, T a b_{\lambda}, T_{\{i\}}, Y_{i}(T),\left.x\right|_{V_{i}}, \mathcal{V}_{x}^{T}, \mathcal{F}_{T}=\overline{\mathcal{V}_{x}^{T}},\left\{\mathcal{F}_{T}\right\}_{T \in T a b_{\lambda}}$

$\S 1.3 \operatorname{col}(i), \tau^{*}(T), T_{i}, \tau^{*}(T),|S|$

$\S 1.4 Z_{x}, S_{n}$, Rank $x, I_{n}, I_{n, k}, \sigma$-basis, $\sigma$-flag, $\sigma_{T}, \mathcal{Z}_{\sigma}, G_{T}$

$\left.\S 2.1 P_{\sigma}, \sigma^{0}, \sigma^{\ell}, \sigma^{r}, c_{\sigma}^{r}((i, j)), c_{\sigma}^{l}((i, j)), c(\sigma)\right),[a, b], b_{\sigma}((i, j)), b(\sigma), b_{\sigma}(f), S_{[a, b]}, \pi_{a, b}(\sigma), R(\sigma)$, $A \leq B, \sigma \geq v, \operatorname{Cov}(a), d_{0}, I_{n, k}^{\max }$

$\S 2.2 \sigma_{\left(i_{1}, j_{1}\right), \ldots,\left(i_{s}, j_{s}\right)}^{-}, \sigma(i, j)=(i, j) \sigma, \mathcal{V}_{G_{\sigma}}, \mathcal{E}_{G_{\sigma}}, a_{\sigma, v}, \mathcal{T}_{F}(\mathcal{V})$

$\S 3.1 \operatorname{Sing}(\sigma)$

$\S 3.2 \sigma_{+a},\left(s_{1}, s_{2}\right)$-maximal completion, $\sigma v_{+n}$

$\S 4.2 \widehat{\sigma}, G r_{d}(n), \mathcal{H}_{x}, \mathcal{B}_{n}=\left\{e_{i}\right\}_{i=1}^{n}, x * y\left(e_{i}\right)$

$\S 5 \quad \operatorname{con}(k)$ 


\section{References}

[1] L. Fresse, Singular components of Springer fibers in the two-column case, Ann. Inst. Fourier 59 (2009), 2429-2444.

[2] L. Fresse, On the singular locus of certain subvarieties of Springer fibers, Math. Res. Lett. 19 (2012) 753-768.

[3] L. Fresse and A. Melnikov, On the singularity of the irreducible components of a Springer fiber in $\operatorname{sl}(n)$, Selecta Math. (N.S.) 16:3 (2010) 393-418.

[4] L. Fresse and A. Melnikov, Some charcterizations of singular components of Springer fibers in the two-column case, J. Algebras and Representation Theory 14 (2011) 1063-1086

[5] L. Fresse, A. Melnikov, S. Sakas-Obeid, On the structure of smooth components of Springer fibers, Proc. of AMS 143 (2015) 2301-2315.

[6] W. Fulton, Young Tableaux with applications to representation theory and geometry, Cambridge Univ. Press, London, 1997.

[7] F.Y.C. Fung, On the topology of components of some Springer fibers and their relation to Kazhdan-Lusztig theory, Adv. Math 178:2 (2003) 244-276.

[8] N. Perrin and E. Smirnov, Springer fiber components in the two column case for type $A$ and $D$ are normal, Bulletin de la SMF 140:3 (2012), 309-333.

[9] V. Lakshmibai and C.S. Seshadri, Singular locus of a Schubert variety, Bull. Amer. Math. Soc. (N.S.) 11:2 (1984), 363-366.

[10] A. Melnikov, B-orbits of nilpotent order 2 and link patterns, Indag. Math.(N.S.) 24, (2013), 443-473.

[11] N. Spaltenstein, On the fixed point set of a unipotent element on the variety of Borel subgroups, Topology 16:2 (1977), 203-204.

[12] N. Spaltenstein, Classes unipotentes et sous-groups de Borel, Lect. Notes Math. 946, Springer-Verlag, Berlin, 1982.

[13] D. A. Timashev, Generalization of the Bruhat decomposition, Russian Acad. Sci. Izv. Math. 45 (1995), 339-352.

[14] J. Tymoczko, The geometry and combinatorics of Springer fibers, in Around Langlands correspondences, Contemp. Math. 691, Amer. Math. Soc., Providence, RI, 2017, 359-376. 